\title{
Coherent vortex simulation of three-dimensional turbulent mixing layers using orthogonal wavelets
}

\author{
By KAI SCHNEIDER ${ }^{1,2}$, MARIE FAR GE ${ }^{3}$, \\ GIULIO PELLEGRINO ${ }^{1,4}$ AND MICHAEL M. ROGERS \\ ${ }^{1}$ LMSNM-GP, CNRS \& Universités d'Aix-Marseille, 38 rue Joliot-Curie, \\ 13451 Marseille Cedex 20, France \\ ${ }^{2}$ CMI, Université de Provence, 39 rue Joliot-Curie, 13453 Marseille Cedex 13, France \\ ${ }^{3}$ LMD-CNRS, Ecole Normale Supérieure, 24 rue Lhomond, 75231 Paris Cedex 05, France \\ ${ }^{4}$ ICT, Universität Karlsruhe (TH), Kaiserstrasse 12, 76128 Karlsruhe, Germany \\ ${ }^{5}$ NASA Ames Research Center, Moffett Field, CA 94035, USA
}

(Received 2 January 2003 and in revised form 9 December 2004)

This paper assesses the potential of coherent vortex simulation (CVS) to compute three-dimensional turbulent mixing layers. CVS splits each turbulent flow realization into two orthogonal parts, one corresponding to coherent vortices which are kept, and the other to an incoherent background flow which is discarded. The CVS filter is applied to data from direct numerical simulations (DNS) of three-dimensional forced and unforced time-developing turbulent mixing layers. The coherent flow is represented by few wavelet modes, but these are sufficient to reproduce the vorticity probability distribution function and the energy spectrum out to the high-wavenumber end of the inertial range. The discarded incoherent background flow is homogeneous, small-amplitude and decorrelated. The CVS-filtering results are then compared with those obtained for the same compression ratio using Fourier low-pass filtering, as employed in large-eddy simulation (LES). Compared to the incoherent background flow of CVS filtering, the subgrid scales of LES filtering are less homogeneous, have much larger amplitude, and exhibit spatial correlations that makes modelling them a difficult challenge. Finally we present simulations of a time-developing mixing layer where the CVS filter is applied at each time step. The results show that CVS preserves the nonlinear dynamics of the flow, and that discarding the incoherent modes is sufficient to model turbulent dissipation.

\section{Introduction}

Turbulent solutions of the Navier-Stokes equations exhibit coherent vortices whose nonlinear interactions control the flow evolution. Since these vortices are localized and excited on a wide range of scales, we have proposed using the wavelet representation of the vorticity field to extract them in two- and three-dimensional homogeneous turbulent flows (Farge, Schneider \& Kevlahan 1999; Farge, Pellegrino \& Schneider 2001). Orthogonal wavelet bases are well suited for this, because they are made of self-similar functions localized in both physical and spectral space (Farge 1992; Daubechies 1992). In Farge et al. (1999) we have introduced a new method to compute turbulent flows, called coherent vortex simulation (CVS). It is based on the 
wavelet-filtered Navier-Stokes equations, whose solutions are computed on an adaptive wavelet basis where wavelets are dynamically selected to track the flow evolution with a reduced number of modes (Schneider \& Farge 2000; Farge \& Schneider 2001; Schneider \& Farge 2002). The success of this methodology hinges on the ability of the wavelets to achieve a significant reduction in the number of modes needed to describe the flow evolution. Prior to attempting the simulation of turbulent flows using CVS, it is necessary to check that wavelets are capable of representing the complexity of a turbulent flow field with fewer modes than conventional basis functions used in the simulation of turbulence. To confirm this, existing direct numerical simulations (DNS) of turbulence are analysed to determine the number of wavelets required to represent the significant features of the flow. We also perform new simulations where the CVS filter is applied at each time step to verify that the CVS filter preserves the nonlinear dynamics of turbulent flows.

In this paper we focus on a prototypical free shear flow, the time-developing turbulent mixing layer. A similar study for homogeneous isotropic turbulence has been made by Farge et al. (2003). The mixing layers examined here are inhomogeneous, with the turbulence statistics varying in the cross-stream direction. Despite being turbulent, they typically contain vestiges of structures similar to those arising from the instabilities of laminar and transitional mixing layers (Brown \& Roshko 1974). Spanwise, roughly two-dimensional rolls of vorticity are separated by braid regions containing streamwise rib vortices, which are roughly aligned with the stretching induced by the spanwise rolls. The dynamical significance of these structures has been the topic of many papers. For a review we refer the reader to Ho \& Huerre (1984).

Turbulent mixing layers are sensitive to their initial conditions. Even if the freestream velocities are the same, the layer spreading rate, the prominence of the organized large-scale structures, and the mixing efficiency of the layer all depend on the details of the initial conditions, such as the character of the flow upstream of the confluence of the two streams, vortical disturbances in the flow, and free-stream disturbances. The time-developing mixing layer simulations of Rogers \& Moser (1994) include three cases with varying levels of two-dimensional forcing applied at the inception of the layer. These flows in turn exhibit varying amounts of organized large-scale structure. In the absence of two-dimensional forcing, such structures are less prominent. Although spanwise rolls are still apparent, the braid regions between them have significant vorticity and large-scale streamwise rib vortices do not persist once the layer is evolving self-similarly. Strong forcing, on the other hand, induces much more organization and rib vortices are observed between the well-defined rolls. The mixing layer flow fields thus provide a good test bed for the extraction of organized structure by wavelet-based CVS filtering. The strongly forced mixing layer has well-organized, large-scale structures that facilitate assessment of whether the wavelets can represent them with fewer modes than alternative descriptions. The unforced mixing layer results will determine whether wavelets can still accomplish the same thing in the absence of such a high level of flow organization.

In the present paper we apply a vector-valued wavelet algorithm to extract coherent vortices from DNS data of two time-developing three-dimensional turbulent mixing layers previously computed by Rogers \& Moser (1994). Section 2 presents the waveletbased CVS-filtering method for extracting coherent vortices out of three-dimensional turbulent flows. Section 3 covers the application of this method to DNS data of a strongly forced and an unforced turbulent mixing layer. In $\S 4$ the results are compared with those obtained for the same compression ratio using a Fourier low-pass filter, as typically used for large-eddy simulation (LES). In $\S 5$ we study the influence of the 
CVS filter on the dynamics of the flow, by performing time integrations of the filtered fields. We compare the results with a fully resolved DNS and two under-resolved DNS, using a similar number of modes as retained by the CVS. In $\S 6$ we compute the time evolution of a turbulent mixing layer by retaining at each time step only the coherent vortices, discarding the incoherent background flow to model turbulent dissipation. The results thus obtained are compared with DNS and confirm that the CVS-filtered simulation fully preserves the nonlinear dynamics. Finally, conclusions are drawn and the prospects of applying CVS to the computation of three-dimensional turbulent flows are discussed in $\S 7$.

\section{Wavelet method for coherent vortex extraction}

In Farge et al. $(1999,2001)$ a wavelet-based method to extract coherent vortices from both two- and three-dimensional turbulent flows was proposed. The algorithm for the three-dimensional case is described below. We consider the vorticity field $\boldsymbol{\omega}(\boldsymbol{x})=\nabla \times \boldsymbol{V}$, computed at resolution $N=2^{3 J}, N$ being the number of grid points and $J$ the corresponding number of octaves. Each component is developed into an orthogonal wavelet series, from the largest scale $l_{\max }=2^{0}$ to the smallest scale $l_{\text {min }}=2^{1-J}$, using a three-dimensional multi-resolution analysis (MRA) (Daubechies 1992; Farge 1992):

$$
\omega(\boldsymbol{x})=\bar{\omega}_{0,0,0,0} \phi_{0,0,0,0}(\boldsymbol{x})+\sum_{j=0}^{J-1} \sum_{i_{x}=0}^{2^{j}-1} \sum_{i_{y}=0}^{2^{j}-1} \sum_{i_{z}=0}^{2^{j}-1} \sum_{\mu=1}^{7} \widetilde{\omega}_{j, i_{x}, i_{y}, i_{z}}^{\mu} \psi_{j, i_{x}, i_{y}, i_{z}}^{\mu}(\boldsymbol{x}),
$$

with

and

$$
\phi_{j, i_{x}, i_{y}, i_{z}}(x)=\phi_{j, i_{x}}(x) \phi_{j, i_{y}}(y) \phi_{j, i_{z}}(z)
$$

$$
\psi_{j, i_{x}, i_{y}, i_{z}}^{\mu}(x)= \begin{cases}\psi_{j, i_{x}}(x) \phi_{j, i_{y}}(y) \phi_{j, i_{z}}(z), & \mu=1, \\ \phi_{j, i_{x}}(x) \psi_{j, i_{y}}(y) \phi_{j, i_{z}}(z), & \mu=2, \\ \phi_{j, i_{x}}(x) \phi_{j, i_{y}}(y) \psi_{j, i_{z}}(z), & \mu=3, \\ \psi_{j, i_{x}}(x) \phi_{j, i_{y}}(y) \psi_{j, i_{z}}(z), & \mu=4, \\ \psi_{j, i_{x}}(x) \psi_{j, i_{y}}(y) \phi_{j, i_{z}}(z), & \mu=5, \\ \phi_{j, i_{x}}(x) \psi_{j, i_{y}}(y) \psi_{j, i_{z}}(z), & \mu=6, \\ \psi_{j, i_{x}}(x) \psi_{j, i_{y}}(y) \psi_{j, i_{z}}(z), & \mu=7,\end{cases}
$$

where $\phi_{j, i}$ and $\psi_{j, i}$ are the one-dimensional scaling function and the corresponding wavelet, respectively. Due to orthogonality, the scaling coefficients are given by $\bar{\omega}_{0,0,0,0}=\left\langle\omega, \phi_{0,0,0,0}\right\rangle$ and the wavelet coefficients by $\widetilde{\omega}_{j, i_{x}, i_{y}, i_{z}}^{\mu}=\left\langle\omega, \psi_{j, i_{x}, i_{y}, i_{z}}^{\mu}\right\rangle$, where $\langle\cdot, \cdot\rangle$ denotes the $L^{2}$-inner product and $\mu$ corresponds to seven discrete directions in three dimensions (from one corner of a cube to the other seven corners).

The extraction algorithm can be summarized as follows:

(i) given $\boldsymbol{\omega}(\boldsymbol{x})$, sampled on a grid $\left(x_{i}, y_{j}, z_{k}\right)$ for $i, j, k=0, N-1$, and the total enstrophy $Z=\frac{1}{2} \int|\omega|^{2} \mathrm{~d} \boldsymbol{x}$,

(ii) perform the three-dimensional wavelet decomposition by applying the Fast Wavelet Transform (FWT) to each component of $\boldsymbol{\omega}$ to obtain the three components of $\widetilde{\omega}_{j, i_{x}, i_{y}, i_{z}}^{\mu}$ for $j=0, J-1, i_{x}, i_{y}, i_{z}=0,2^{J-1}-1$, and $\mu=1, \ldots, 7$,

(iii) compute the threshold

$$
\epsilon_{T}=\sqrt{\frac{4 Z}{3} \ln N}
$$



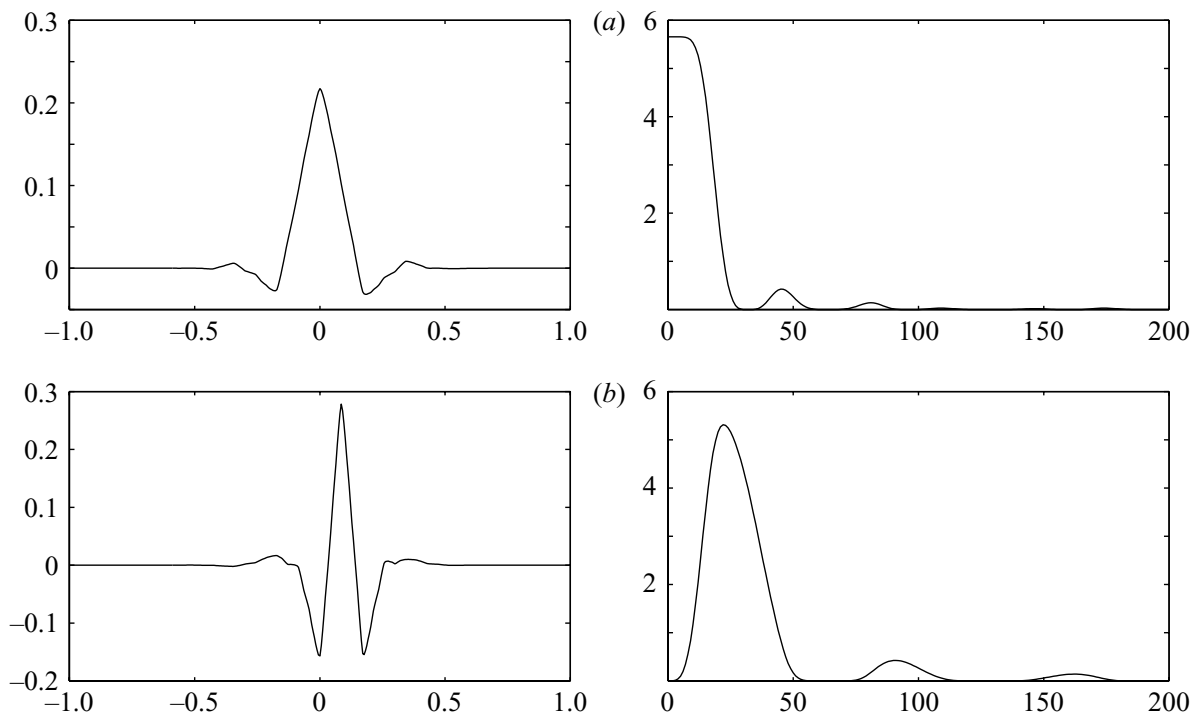

Figure 1. Coiflet 12 wavelet. (a) Scaling function $\phi$ in physical space (left) and spectral space (right). (b) Wavelet $\psi$ in physical space (left) and spectral space (right).

and split the coefficients $\widetilde{\boldsymbol{\omega}}$ into

$$
\widetilde{\boldsymbol{\omega}}_{C}=\left\{\begin{array}{ll}
\widetilde{\boldsymbol{\omega}} & \text { for }|\widetilde{\boldsymbol{\omega}}|>\epsilon_{T} \\
0 & \text { otherwise }
\end{array} \quad \widetilde{\boldsymbol{\omega}}_{I}= \begin{cases}\widetilde{\boldsymbol{\omega}} & \text { for }|\widetilde{\boldsymbol{\omega}}| \leqslant \epsilon_{T} \\
0 & \text { otherwise }\end{cases}\right.
$$

(iv) perform the three-dimensional wavelet reconstruction by applying the inverse FWT to compute $\omega_{C}$ and $\omega_{I}$ from $\widetilde{\omega}_{C}$ and $\widetilde{\boldsymbol{\omega}}_{I}$, respectively,

(v) use Biot-Savart's relation

$$
\boldsymbol{V}=(\nabla \times)^{-1} \boldsymbol{\omega}
$$

to reconstruct the coherent and incoherent velocity fields from the coherent and incoherent vorticity fields, respectively.

The above vortex extraction algorithm is of linear complexity, as the FWT requires $O(N)$ operations, where $N$ denotes the total number of grid points. For the decomposition one FWT is performed for each component of the vorticity vector $\omega$. To reconstruct the coherent vorticity $\omega_{C}$ in physical space one inverse FWT is required for each component. The incoherent vorticity vector $\omega_{I}$ is obtained by taking the difference between the total and the coherent vorticity in physical space. This yields in total six FWTs. The wavelets used are orthogonal Coiflets (figure 1) of order $c=12$ having four vanishing moments, where $c$ denotes the length of the associated quadratic mirror filter (Daubechies 1992; Farge 1992). As a result, the operation count of the whole algorithm is $6 \mathrm{Nc}$ compared with $6 N \log _{2} N$ for the standard fast Fourier transform. Therefore the FWT becomes faster than the FFT for $N>2^{c}$.

Note that the threshold $\epsilon_{T}$ only depends on the total enstrophy $Z$ and on the number of grid points $N$, without any adjustable parameters. The choice of this threshold (equation (2.3)) is based on theorems (Donoho 1993; Donoho \& Johnstone 1994) proving optimality of the wavelet representation to denoise signals in the presence of Gaussian white noise, since this wavelet-based estimator minimizes the maximal $L^{2}$-error for functions with inhomogeneous regularity. 
The two vorticity fields thus obtained, $\omega_{C}$ and $\omega_{I}$, are orthogonal, which ensures a separation of the total enstrophy into $Z=Z_{C}+Z_{I}$ because the interaction term $\left\langle\boldsymbol{\omega}_{C}, \boldsymbol{\omega}_{I}\right\rangle$ vanishes. For the energy $E$ the interaction term $\left\langle\boldsymbol{V}_{C}, \boldsymbol{V}_{I}\right\rangle$ does not vanish, as the wavelets used here are not eigenmodes of the inverse curl operator, but it does remain negligible, less than $1 \% E$ (Farge et al. 2001).

As the orthogonal wavelet transform does not commute with the divergence operator and the vector-valued wavelet basis is not divergence-free, i.e. $\nabla \cdot \psi_{\lambda} \neq 0$, the CVS filtering does not yield coherent and incoherent vorticity that are perfectly divergence-free. There are several possible ways to ensure that the coherent vorticity remains divergence-free:

(i) use divergence-free orthogonal wavelets (Lemarié 1992),

(ii) decompose $\boldsymbol{\omega}$ into $\boldsymbol{\omega}=\boldsymbol{\omega}_{d i v}=0+\nabla \phi$; then $\phi$ can be calculated by taking the divergence which leads to a Poisson equation $\nabla^{2} \phi=\nabla \cdot \omega$.

(iii) apply the previous decomposition, not to the solution, but to the wavelet basis itself, which can be done as a precalculation since the wavelet decomposition is a linear transformation.

However, this is not a key issue in practice, since the divergent component of the decomposed vorticity field remains less than $3 \%$ of the total enstrophy and appears mostly in the dissipation range (Farge et al. 2003). The same problem is also encountered for vortex methods applied to three-dimensional turbulent flows (Winckelmans 1995). Note that the corresponding velocity fields are divergence-free because they have been reconstructed using the Biot-Savart relation.

\section{Application to mixing layers}

Using compactly supported Coiflet 12 wavelets (Daubechies 1992), the above algorithm is applied to two instantaneous three-dimensional time-developing turbulent mixing layer flow fields previously generated by Rogers \& Moser (1994). Both flows are evolving self-similarly at the times examined and the Taylor-microscale Reynolds number $R_{\lambda}$ is about 150 . The first flow field is the strongly forced mixing layer at time $\tau=t \Delta U / \delta_{m}^{0}=101.5$, where $\Delta U$ is the velocity difference across the layer and $\delta_{m}^{0}$ is the initial layer momentum thickness. The second flow field is the unforced simulation at time $\tau=150.0$. Flow visualization of both these flow fields is given in Rogers \& Moser (1994). As noted in the introduction, the forced flow exhibits much more large-scale organization than the unforced flow. The coordinate axes are defined as $O x$ for the streamwise direction, $O y$ for the cross-stream (vertical) direction and $O z$ for the spanwise direction.

The orthogonal wavelet decomposition used here requires that the number of computational grid points in each of the coordinate directions be a power of 2 . At $\tau=101.5$, the forced mixing layer flow field was computed using $384 \times 120 \times 128$ modes. Thus, prior to performing the wavelet filtering, the vorticity field was interpolated onto a finer physical-space grid with $512 \times 256 \times 128$ points. The unforced mixing layer flow field, computed using $512 \times 180 \times 192$ modes, was also interpolated onto a $512 \times 256 \times 128$ physical-space mesh (truncated spanwise representation). Owing to memory limitations of the graphics package used for three-dimensional flow visualization, both vorticity fields were reduced to $64^{3}$ for flow visualization.

\subsection{Forced mixing layer}

Figure 2(a) shows the modulus of vorticity for the total flow in the forced case. Clearly evident are four transverse 'rolls', produced by the two-dimensional Kelvin-Helmholtz 

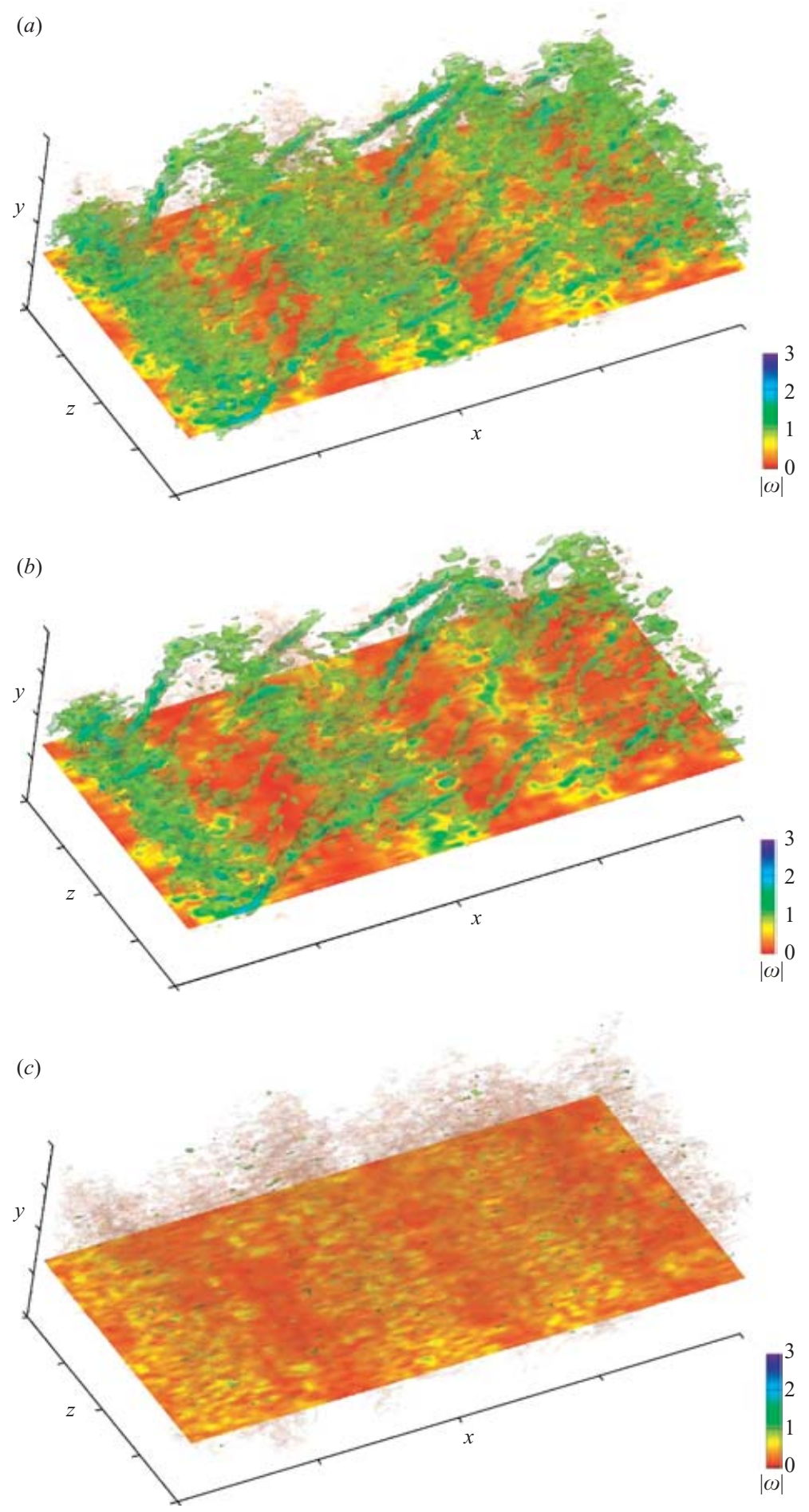

FIGURE 2. Forced mixing layer: (a) modulus of the total vorticity of the forced threedimensional mixing layer; $(b)$ modulus of the coherent vorticity; $(c)$ modulus of the incoherent vorticity. The same colour map is used for the three fields. 

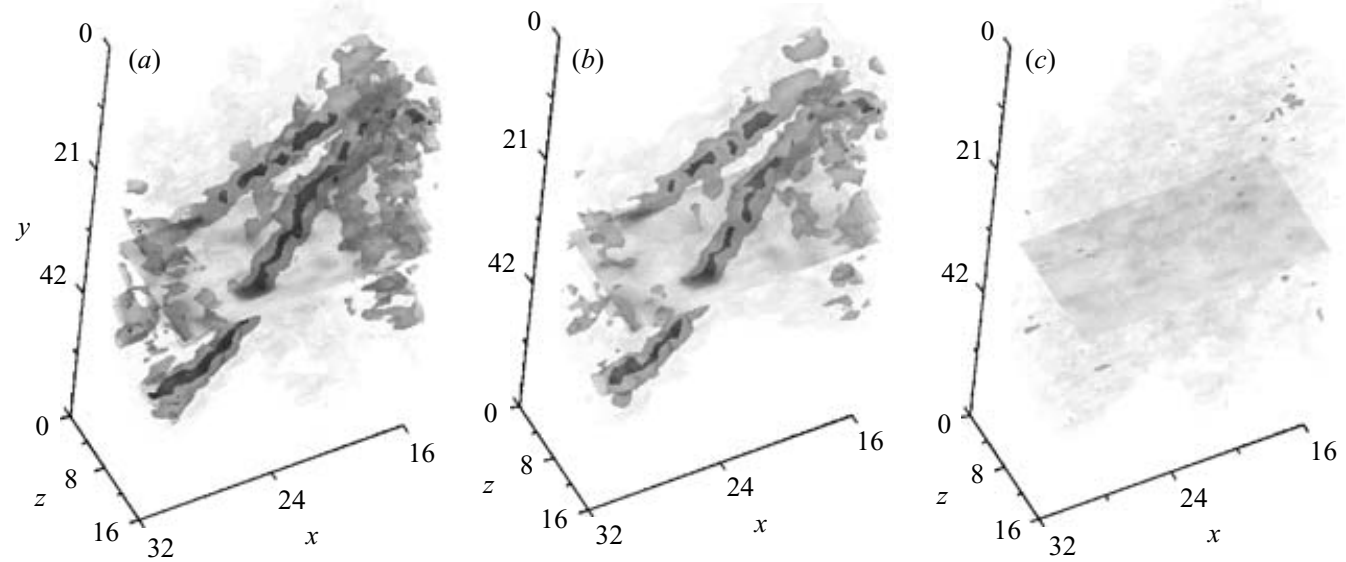

FiguRE 3. Zoom of figures $2(a)$ and $2(b)$. Iso-surfaces of vorticity modulus for $(a)$ total flow, $(b)$ coherent part, $(c)$ incoherent part using the same colour map as in figures $2(a)$ and 2(b).

instability, and pronounced longitudinal vortex tubes, called 'ribs', which result from a three-dimensional instability.

The coherent vorticity (figure $2 b$ ), which is reconstructed using only $3.8 \%$ of the wavelets, captures most of the turbulent kinetic energy $(99 \%)$ and enstrophy $(83 \%)$, for all wavenumbers associated with the large scales and the inertial range (figure $4 a$ ). The same transverse rolls and longitudinal ribs present in the total vorticity field (figure $2 a$ ) are retained in the coherent vorticity field (figure $2 b$ ).

In figure 3 we zoom in on a pair of 'rib' vortices visible in figure $2(a, b)$. This figure confirms that the coherent flow contains the vortex tubes present in the total flow, including even the smaller ones such as the ribs, whereas the incoherent flow is structureless.

To confirm this, we observe that the probability distribution function (PDF) of the coherent vorticity is similar to that of the total vorticity, even for the extreme values (figure $4 c$ ). In contrast, the incoherent vorticity (figure $2 c$ ), which is represented by $96.2 \%$ of the wavelets, contains less than $1 \%$ of the turbulent kinetic energy and $17 \%$ of the enstrophy. It is structureless and quasi-homogeneous with low amplitude. The PDF of the incoherent vorticity (figure $4 c$ ) decays exponentially, with much reduced extreme values compared to the PDF of the total vorticity.

The similarity between the streamwise one-dimensional energy spectra (figure $4 a$ ) of the coherent and the total flow indicates that the energetic turbulent motions are well captured by the CVS filtering for all except the highest wavenumbers, where dissipation dominates. In contrast, the incoherent part contains very little energy ( $1 \%$ of the total) and is uncorrelated in space, i.e. the energy spectrum is flat at all scales, corresponding to an energy equipartition. The incoherent energy dominates the coherent energy only in the highest wavenumbers, where dissipation takes over.

The cross-stream profiles of the spanwise vorticity component averaged in the streamwise and spanwise directions $\overline{\omega_{z}}$ are shown in figure 5 for the total, coherent, and incoherent flow. They show that the coherent vorticity exactly follows the total vorticity, while the incoherent vorticity oscillates weakly around zero, with an amplitude of less than $4 \%$ of the maximal value of $\left|\overline{\omega_{z}}\right|$, which is the maximal vorticity of the primary rolls. This quantifies the similarity between the coherent and total vorticity and confirms that the coherent flow retains most of the enstrophy. 

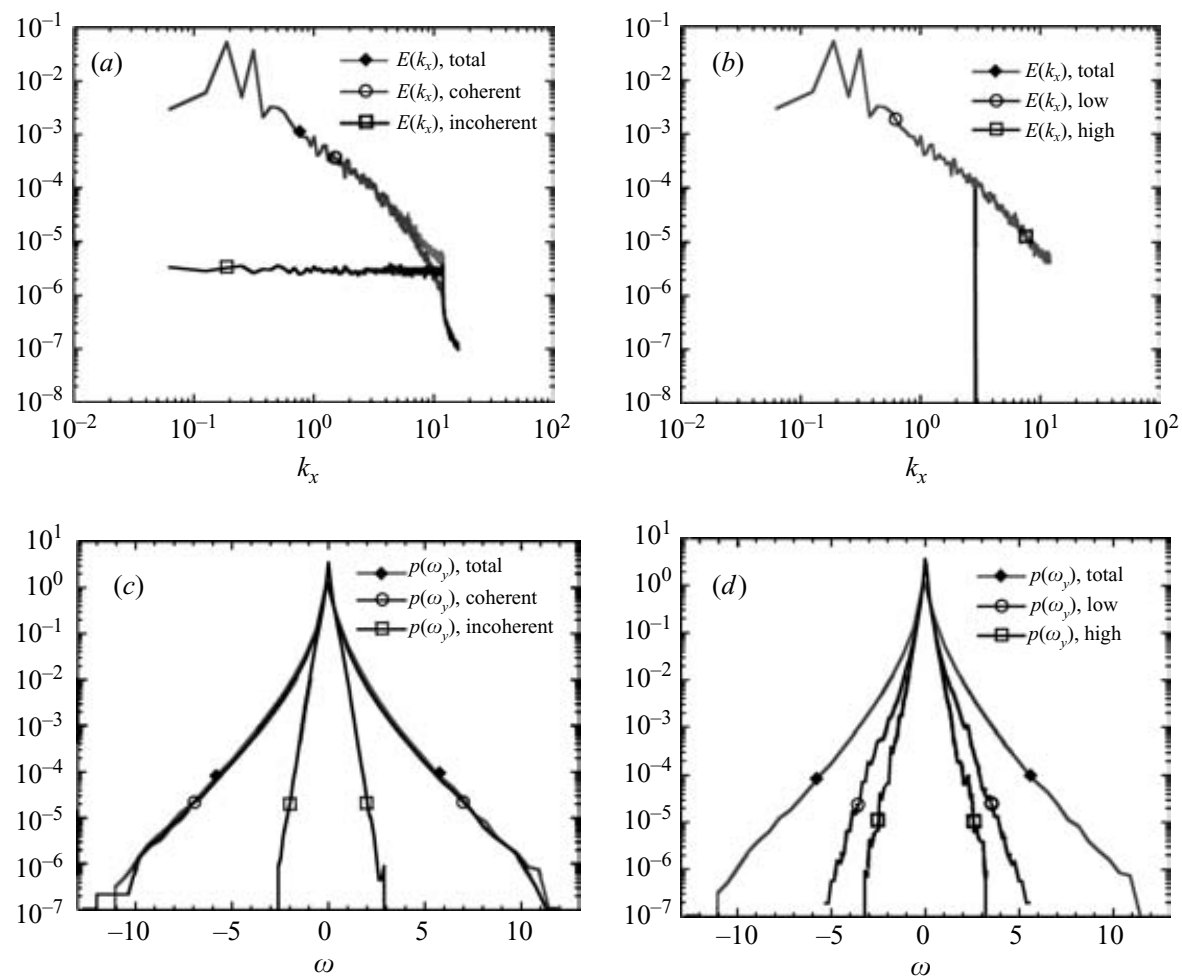

FIgure 4. Comparison of CVS $(a, c)$ with LES $(b, d)$ filtering for the forced mixing layer. Energy spectra in the streamwise direction $(a, b)$ and PDF of vorticity $(c, d)$ of total, coherent, and incoherent flow using CVS filtering $(a, c)$, and of low-wavenumber and high-wavenumber components using LES filtering $(b, d)$.

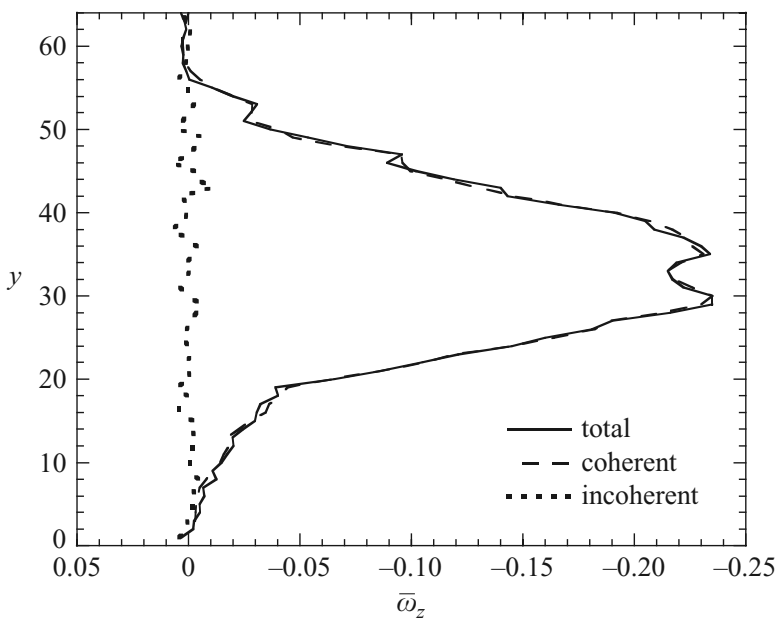

Figure 5. Cross-stream profiles of the spanwise vorticity component averaged in the streamwise and spanwise directions $\overline{\omega_{z}}$, for the total, coherent, and incoherent vorticity of the forced mixing layer. 


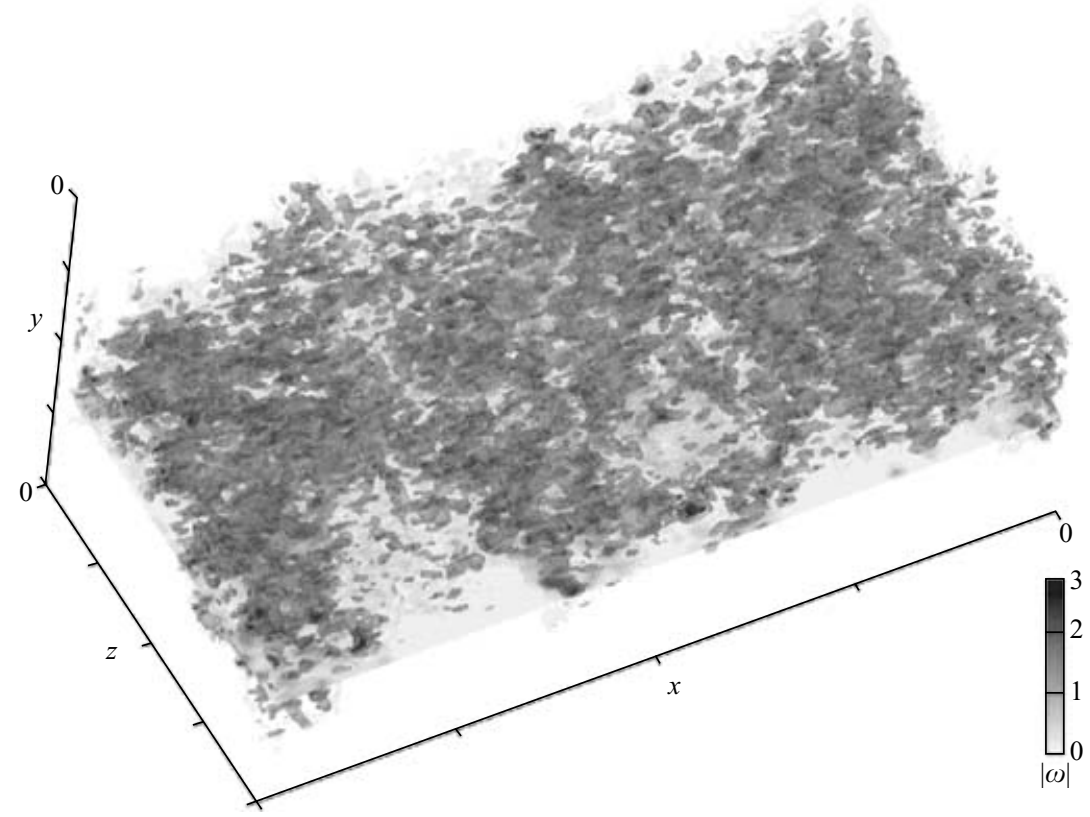

FIGURE 6. Modulus of the total vorticity of the unforced three-dimensional mixing layer.

\subsection{Unforced mixing layer}

Now we consider the unforced mixing layer and apply the CVS filtering to this flow. Figure 6 shows the modulus of vorticity for the total flow. In contrast to the forced mixing layer (figure 1), there is little evidence of organized, large-scale structures in the unforced flow, with less pronounced transverse rolls and no obvious rib vortices.

Despite the lack of large-scale organized structure, the coherent vorticity (figure $7 a$ ), which is represented by only $4.2 \%$ of the wavelet coefficients, still captures the character of the total vorticity. The incoherent vorticity (figure $7 b$ ), represented by $95.8 \%$ of the wavelet coefficients, again appears quasi-homogeneous, with little organized structure and much smaller amplitude.

The validity of the decomposition is further confirmed by the vorticity PDF (figure $8 c$ ). Again, the coherent vorticity presents the same non-Gaussian distribution as the total vorticity, whereas the PDF of the incoherent vorticity follows an exponential decay with a much reduced variance compared to the total vorticity. As for the forced case, the coherent flow has the same spectral distribution of energy as the total flow for all scales up to the dissipative wavenumbers (figure $8 a$ ). In contrast, the incoherent flow is characterized by a small-amplitude, flat energy spectrum corresponding to energy equipartition. The similarity in the number of wavelets required to represent the large-scale organized structures in both flows indicates that prominent large-scale structures are not a necessity for the wavelet decomposition to successfully represent the coherent features of the flow.

\section{Comparison between CVS and LES filtering}

We now consider the results obtained with LES filtering applied to the two timedeveloping mixing layers examined above. Here we choose the simplest LES filter, a low-pass Fourier cut-off filter, and apply it in each of the three coordinate directions. 

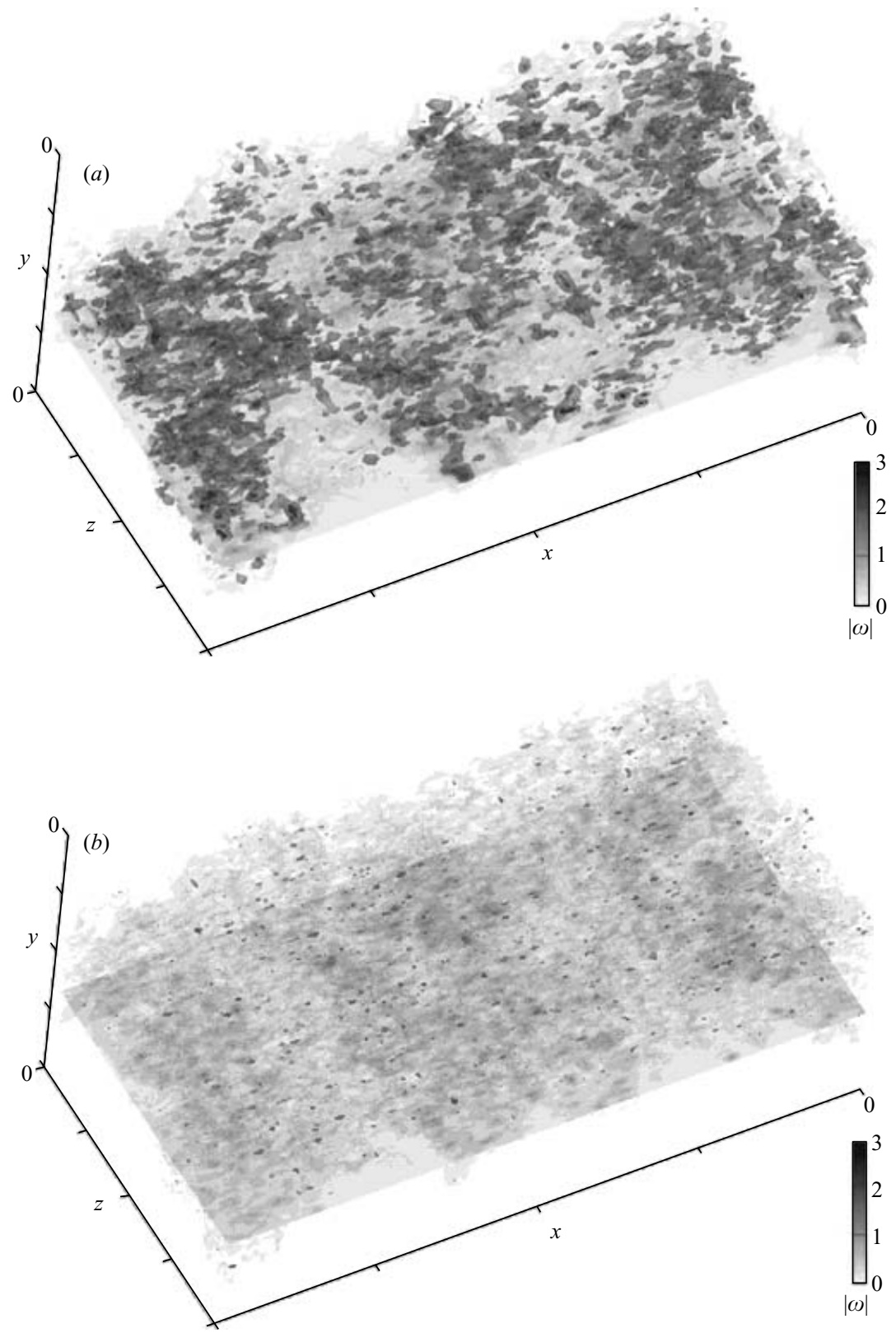

FIGURE 7. Unforced mixing layer: $(a)$ modulus of the coherent vorticity; $(b)$ modulus of the incoherent vorticity. The same colour map is used for both fields.

The resulting filtered flow retains $9 \%$ of the $512 \times 256 \times 128$ modes, or $3.2 \%$ of the original computational Fourier/Jacobi modes for the forced case $(6.3 \%$ for the unforced case). The discarded modes correspond to the subgrid scales of a LES. The amount of information retained in the LES and CVS-filtering schemes is thus comparable for the forced simulation, consisting of at least $3 \%$ of the full dataset, and favours the LES for the unforced flow. 

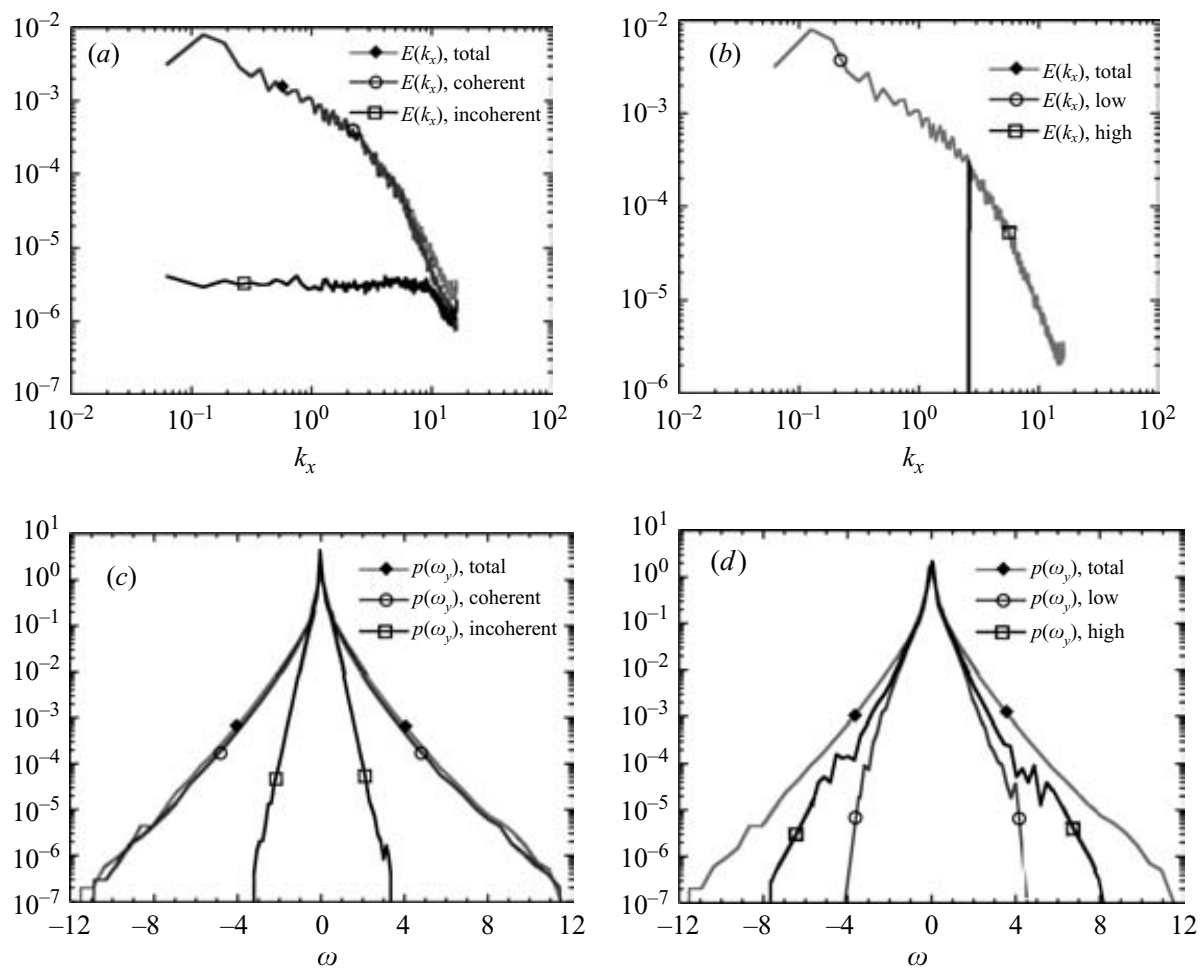

FIgURE 8. Comparison of CVS $(a, c)$ with LES $(b, d)$ filtering for the unforced threedimensional mixing layer, at resolution $N=512 \times 256 \times 128$. Energy spectra in the streamwise direction $(a, b)$ and PDF of vorticity $(c, d)$ of total, coherent, and incoherent flow using CVS filtering $(a, c)$, and of large-scale and subgrid-scale components using LES filtering $(b, d)$.

The LES filtering keeps only the scales larger than that associated with the cutoff wavenumber (figures $4 b$ and $8 b$ ). We observe that the LES filtering smooths the coherent vortices since the extrema of vorticity are strongly reduced (see the PDF on figures $4 d$ and $8 d$ ). In contrast, the CVS filtering retains the organized features without any smoothing, whatever their scale, and, as noted previously, the shape of the vorticity PDF is fully preserved, even for the extreme values of vorticity (figures $4 c$ and $8 c$ ).

The large-scale and subgrid-scale vorticity fields, obtained by LES filtering of the unforced mixing layer, exhibit organized features of similar magnitude (figure 9). Additionally, their vorticity PDFs (figure $8 d$ ) have about the same variation and the large-scale vorticity does not capture the distribution of the total flow. In fact, the subgrid-scale modes, whose effect must be modelled in LES, have a more nonGaussian distribution than the large-scale modes that are resolved by LES. Therefore the subgrid-scale models used for LES should not be based on the assumption of Gaussian behaviour for the subgrid scales.

We now compare and contrast the character of the flow fields discarded by CVS filtering and LES filtering. The CVS filtering extracts the coherent structures from turbulent flows, leaving only white noise for the incoherent flow, which is discarded. The incoherent flow is structureless and strained by the coherent flow, inhibiting nonlinear interaction and preventing the transfer of energy from the incoherent modes to the coherent modes. In contrast, LES filtering discards a subgrid-scale flow 

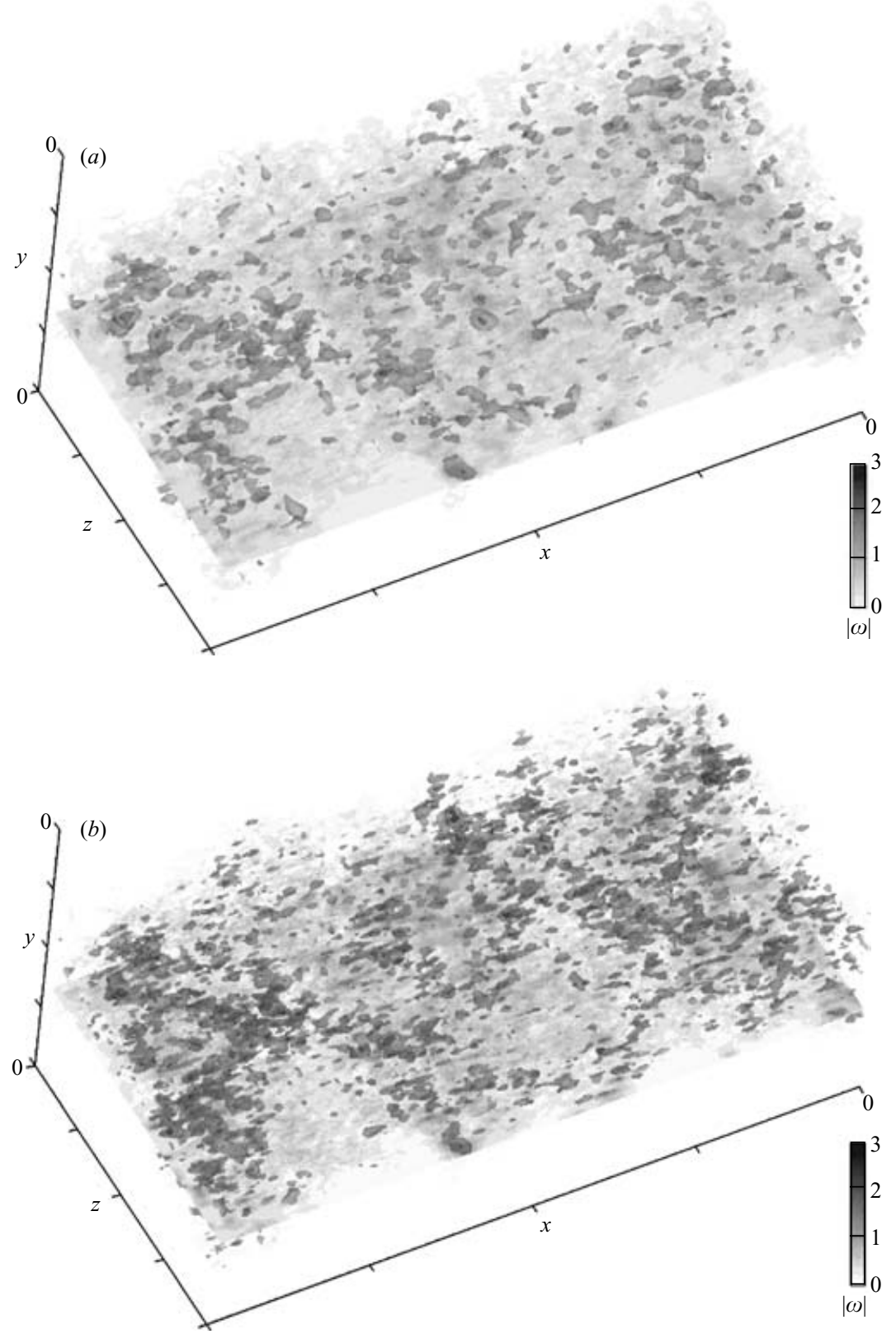

FIGURE 9. Unforced mixing layer: $(a)$ modulus of the large-scale vorticity reconstructed from $9 \%$ of the low-wavenumber Fourier modes; $(b)$ modulus of the subgrid-scale vorticity reconstructed from $91 \%$ of the high-wavenumber Fourier modes. The same colour map is used for both fields.

that has spatial structure. If these subgrid-scale coherent structures were kept, they would interact nonlinearly and transfer energy back to larger scales (backscatter). Moreover, the variability of the total field is not fully retained by the LES filtering as it is by the CVS filtering. As a consequence, the discarded high-wavenumber modes contain more enstrophy than the incoherent modes discarded by the CVS filtering. 


\begin{tabular}{llrrrr}
\hline & & DNS-f & CVS-f & C-DNS1-f & C-DNS2-f \\
& Number of modes $N$ & $100 \%$ & $3.8 \%$ & $8.8 \%$ & $3.7 \%$ \\
Cross-stream-integrated & $E_{C S I}(\tau=101.5)$ & 3.196 & 3.121 & 3.155 & 3.119 \\
$\quad$ energy & $E_{C S I}(\tau=116.4)$ & 3.631 & 3.608 & 3.650 & 3.672 \\
Cross-stream-integrated & $Z_{C S I}(\tau=101.5)$ & 16.832 & 13.669 & 11.616 & 9.280 \\
$\quad$ enstrophy & $Z_{C S I}(\tau=116.4)$ & 16.528 & 15.768 & 16.872 & 15.254 \\
Momentum thickness & $\tau=101.5$ & 1.871 & 1.870 & 1.870 & 1.870 \\
& $\tau=116.4$ & 2.080 & 2.083 & 2.083 & 2.084
\end{tabular}

TABLE 1. Forced mixing layer. Statistics at $\tau=101.5$ and $\tau=116.4$.

For LES, if we do not model the effect of the subgrid-scale modes on the resolved modes, the energy accumulates at the cutoff and the computation may diverge. For CVS, if we do not model the effect of the discarded incoherent modes on the resolved coherent modes, there is no risk of divergence since energy can be transferred in both directions throughout the fully resolved inertial range. Discarding the incoherent modes corresponds to turbulent dissipation, because these modes are structureless, homogeneous, and uncorrelated.

\section{Dynamical analyses of the filtered fields}

To better understand the effect of the CVS filtering on the dynamics of the flow, the CVS-filtered flows have been advanced in time using the DNS code of Rogers $\&$ Moser (1994). These flow evolutions are then compared with those of the original DNS calculations to ascertain any differences in flow development. Similarly, the LES-filtered fields are also advanced in time with the same code but using coarser resolutions, comparable to the number of modes retained by the CVS filtering. This code has no explicit subgrid-scale model, so these latter simulations are effectively coarse DNS, which are denoted C-DNS.

\subsection{Forced mixing layer}

In the forced case we perform time integrations from $\tau=101.5$ to $\tau=116.4$, using as an inititial condition either the total flow, or the CVS-filtered flow, or two LES-filtered flows of different compressions. For the CVS-filtered flow (CVS-f) we integrated the coherent part, which corresponds to $3.85 \%$ of the total number of modes $(N=512 \times 256 \times 128)$. A total of 411 steps was required to reach the final time. For the LES-filtered flow we performed two coarse DNS, one retaining $8.79 \%$ of $N$ modes at large scales corresponding to a resolution $192 \times 120 \times 64$ (named C-DNS1-f) and one retaining $3.71 \%$ of $N$ modes corresponding to a resolution $144 \times 90 \times 48$ (named C-DNS2-f). The statistics of the results obtained for the four integrations are displayed in table 1.

We then analyse the time evolution of the cross-stream-integrated enstrophy plotted in figure 10. We observe that for C-DNS1-f and C-DNS2-f the enstrophy level increases with time. In the CVS-f case enstrophy also increases, although more gradually, and it smoothly approaches the DNS-f curve. Note that at the initial time, CVS-f retained more enstrophy than C-DNS1-f and C-DNS2-f. The energy spectra at $\tau=116.4$ are shown in figure 11. For C-DNS1-f and C-DNS2-f energy tends to pile up at the cut-off wavenumbers (cf. the zoom in figure 11) because there is no subgrid-scale model. For CVS-f we find an almost perfect superposition with the 


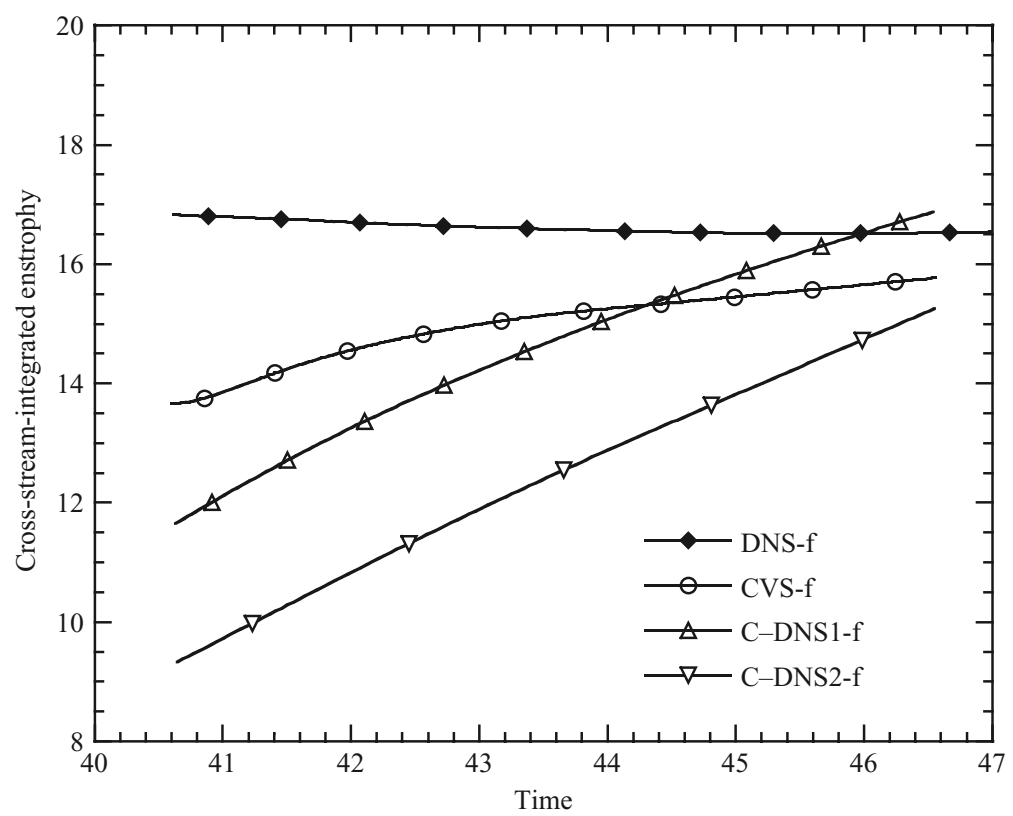

FIGURE 10. Forced mixing layer. Time evolution of the cross-stream-integrated enstrophy.

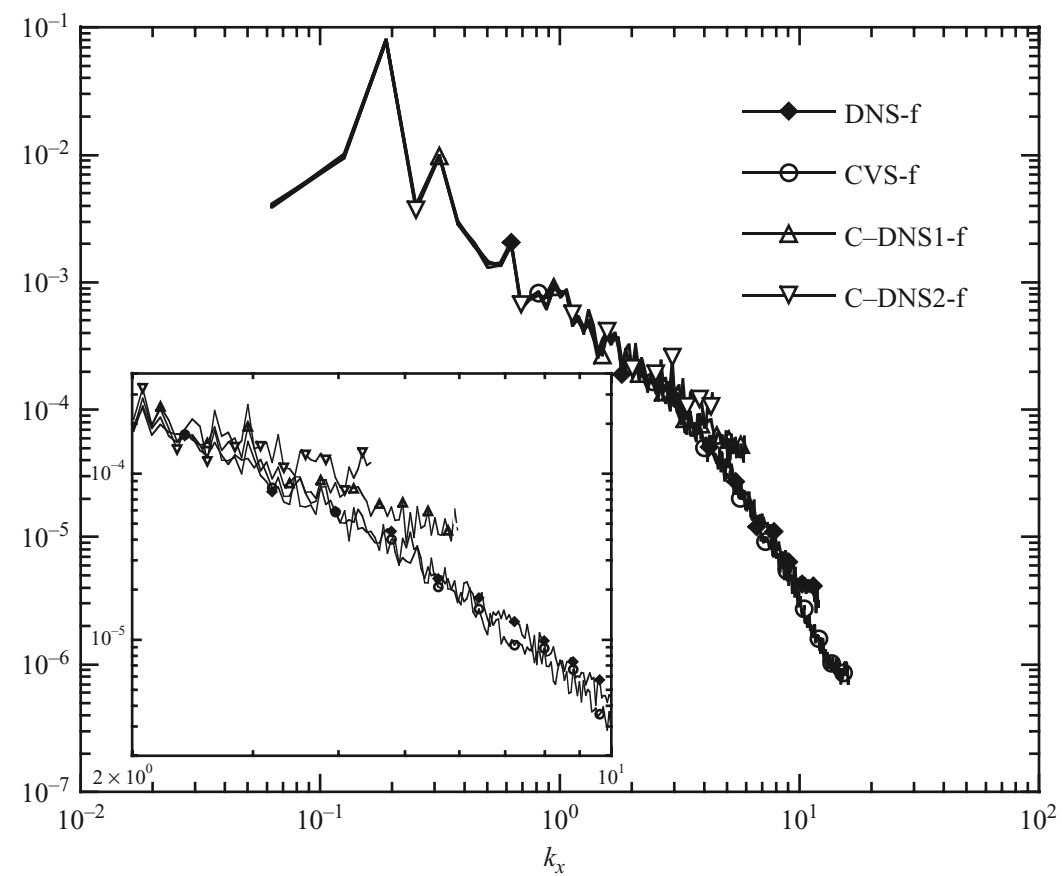

FIGURE 11. Forced mixing layer. Energy spectra in the streamwise direction at $\tau=116.4$.

DNS simulation. Additionally the PDF of the vertical vorticity $\left(\omega_{y}\right)$ at time $\tau=116.4$ shows a better match between the evolved CVS flow and the DNS (figure 12). For both C-DNS1-f and C-DNS2-f the vorticity extrema are reduced in comparison to the DNS. On the other hand, the agreement between the evolution of the CVS 


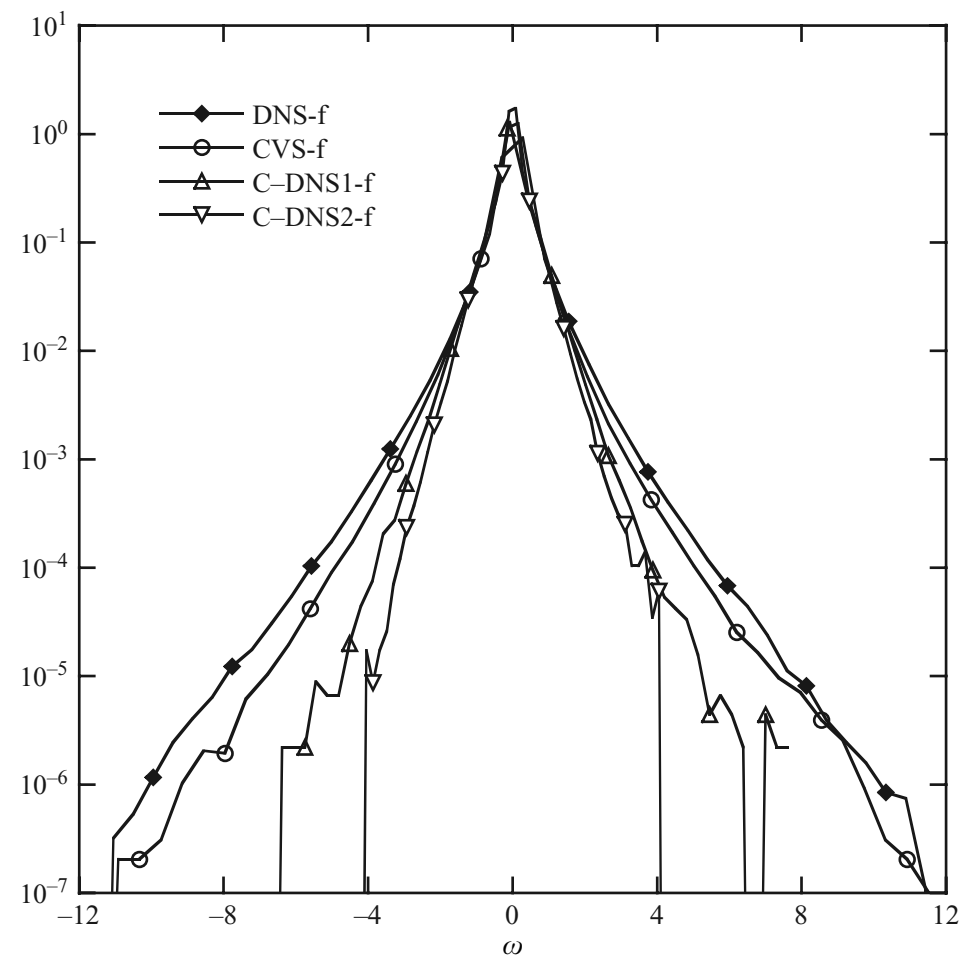

FIGURE 12. Forced mixing layer. PDF of vertical vorticity $\left(\omega_{y}\right)$ at $\tau=116.4$.

flow and the two C-DNS flows for large-scale statistics is much better. All three precisely reproduce the evolution of the momentum thickness (the results differ less than $0.04 \%$, cf. table 1) and the shapes of the mean streamwise velocity profiles at the final time agree perfectly (figure not shown). The evolution of the cross-stream integrated turbulent kinetic energy, also shown in table 1, is likewise similar for all the flows, with the final values in the C-DNS flows being slightly higher. Actually, this discrepancy and the one observed for enstrophy can be largely attributed to the lower resolution of the C-DNS. Thus the large-scale field evolves accurately for all of the filters used, even with coarse resolution. This is perhaps in part due to the forcing, which makes the large-scale two-dimensional motions play an important role in the evolution.

\subsection{Unforced mixing layer}

As with the forced case, we perform time integrations over a fixed time interval from various initial filterings of the unforced flow at $t=150.0$. For the reference case (DNS-u) the total field is used as the initial condition, whereas for the CVS-filtered case (CVS-u) the coherent part from the CVS wavelet filtering, comprised of $4.17 \%$ of the total number of modes $(N=512 \times 256 \times 128)$ is used. This flow was time advanced 388 time steps to $\tau=168.1$ and the resulting flow evolution was compared to that of the full simulation and to a coarse DNS (C-DNS-u) begun from an LES-filtered field with $128 \times 90 \times 48$ modes, or $3.30 \%$ of the modes used to represent the full DNS field. The statistics obtained for the three integrations are assembled in table 2. Again we find in all cases excellent agreement for the momentum thickness and the mean streamwise velocity profile (figures not shown). 


\begin{tabular}{llcrr}
\hline & & DNS-u & CVS-u & C-DNS-u \\
& Number of modes $N$ & $100 \%$ & $4.17 \%$ & $3.30 \%$ \\
Cross-stream-integrated & $E_{C S I}(\tau=150.0)$ & 2.062 & 2.013 & 1.960 \\
$\quad$ energy & $E_{C S I}(\tau=168.1)$ & 2.194 & 2.297 & 2.387 \\
Cross-stream-integrated & $Z_{C S I}(\tau=150.0)$ & 20.217 & 16.503 & 9.952 \\
$\quad$ enstrophy & $Z_{C S I}(\tau=168.1)$ & 19.612 & 19.189 & 18.992 \\
Momentum thickness & $\tau=150.0$ & 1.984 & 1.984 & 1.984 \\
& $\tau=168.1$ & 2.194 & 2.192 & 2.199
\end{tabular}

TABLE 2. Unforced mixing layer. Statistics at $\tau=150.0$ and $\tau=168.1$.

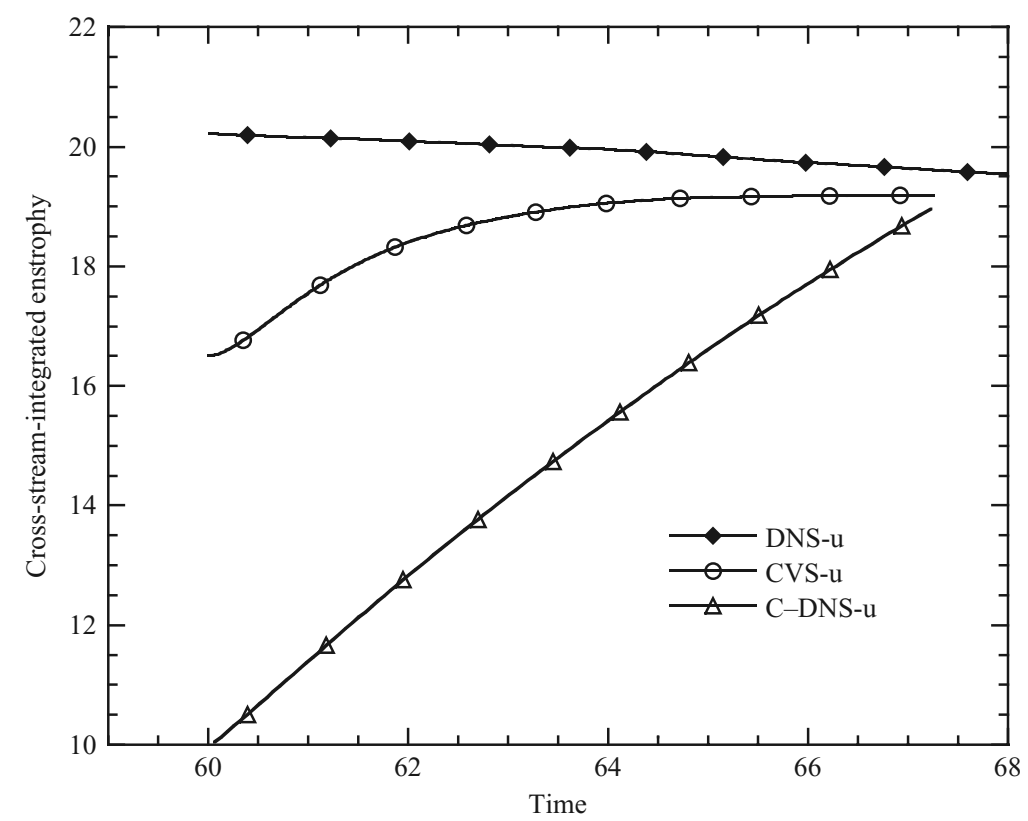

FIGURE 13. Unforced mixing layer. Time evolution of the cross-stream-integrated enstrophy.

The cross-stream integrated enstrophy (figure 13) rapidly shoots up for C-DNS-u, while for CVS-u it gradually approaches the DNS evolution. Conclusions drawn from the energy spectra at $\tau=168.1$ in figure 14 are consistent with those drawn in the forced case, i.e. for C-DNS-u energy tends to pile up at the cut-off wavenumber owing to the lack of a subgrid model, while for CVS-u we find a very good agreement up to the finest scales. The vorticity PDFs at time $\tau=168.1$ (figure 15) show that CVS-u preserves the extrema much better than C-DNS-u, with the PDF for C-DNS-u being much narrower than that for DNS-u.

\section{Coherent vortex simulation}

\subsection{Principle}

In this section we check the ability of the CVS approach to track the flow evolution by computing the time evolution of the coherent flow only, discarding the incoherent enstrophy at each time step. The resulting evolution is compared with that obtained 


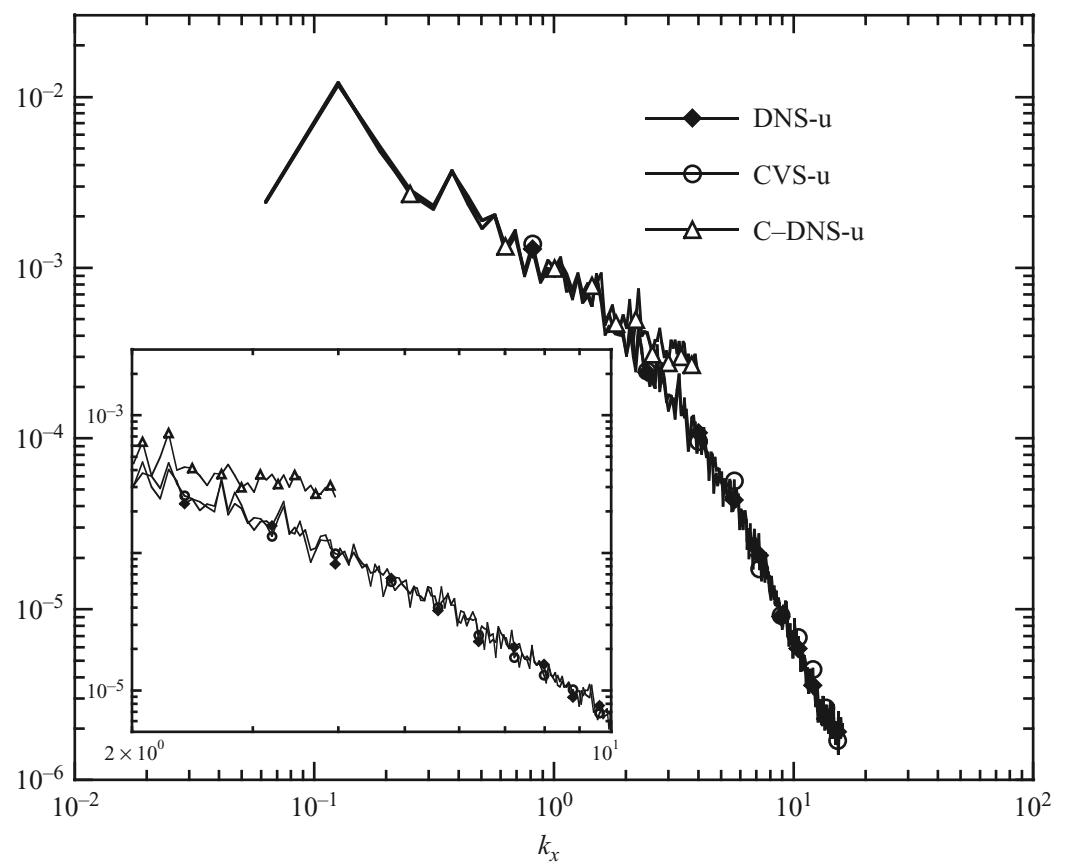

FIGURE 14. Unforced mixing layer. Energy spectra in the streamwise direction at $\tau=168.1$.

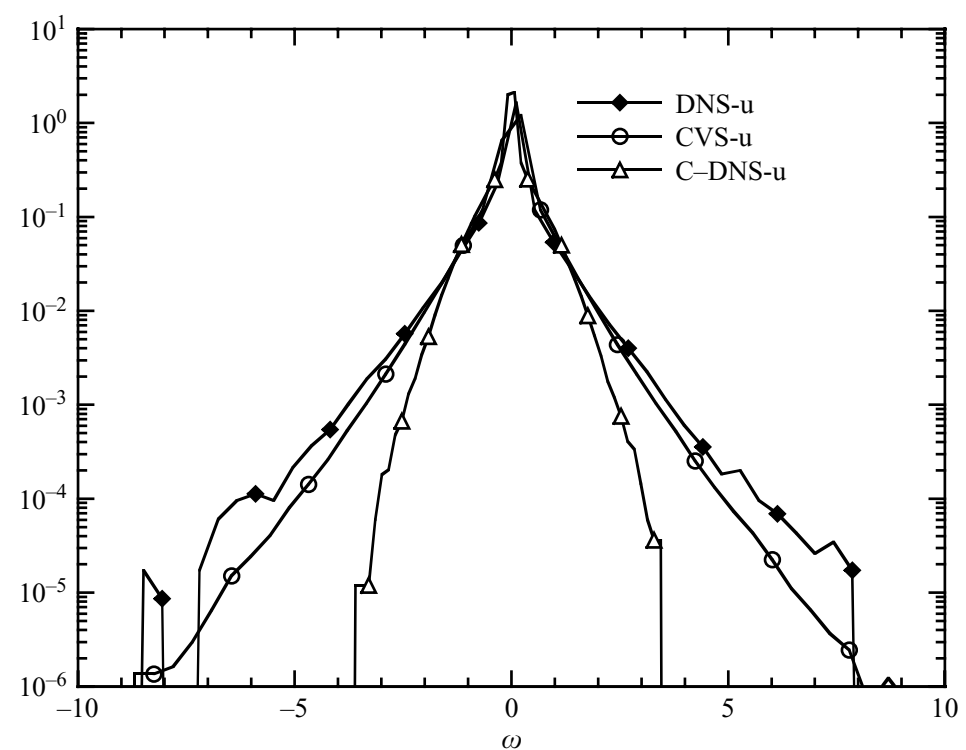

FIGURE 15. Unforced mixing layer. PDF of vertical vorticity $\left(\omega_{y}\right)$ at $\tau=168.1$.

from DNS. For both computations we use a pseudo-spectral code kindly provided by Pierre Comte. The discretization is based on Fourier transforms in the streamwise and spanwise directions, and sine/cosine transforms in the vertical direction. The time integration is done with a low-storage Runge-Kutta scheme of third order. For more details on the numerical code see Comte, Lesieur \& Lamballais (1992). 


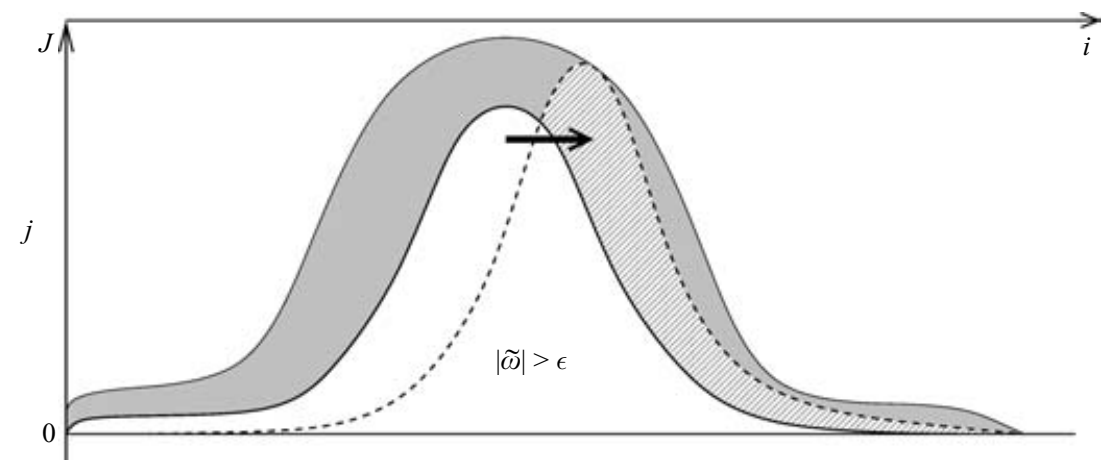

Figure 16. Security zone in wavelet coefficient space ( $i$ position and $j$ scale). A coherent vortex at time $t_{n}$ is represented by only the wavelet coefficients with vorticity modulus $\left|\tilde{\omega}\left(t_{n}\right)\right|$ larger than the threshold $\epsilon_{T}\left(t_{n}\right)$, which corresponds to the filtered wavelet basis at time $t_{n}$ (area below the solid curve). To allow the coherent vortex to translate or be distorded by the Navier-Stokes nonlinear dynamics, a security zone is added (grey area). The adaptive wavelet basis is the union of the filtered wavelet basis and the security zone. The solution is then advanced to time $t_{n+1}$ and is represented by only the wavelet coefficients $\left|\tilde{\omega}\left(t_{n+1}\right)\right|>\epsilon_{T}\left(t_{n+1}\right)$, which correspond to the filtered wavelet basis at time $t_{n+1}$ (area below the dashed curve).

In this code we implemented the CVS filtering algorithm described in $\S 2$. Since it is designed to eliminate the noisy part of the flow, it does not make sense to start filtering at $t=0$, because this would filter out the initial random perturbations needed to trigger the flow instability. Therefore, we begin to apply the CVS filtering at $t=5 \delta_{m}^{0} / \Delta U$. At each time step, the vorticity $\boldsymbol{\omega}$ is computed from the velocity $\boldsymbol{V}$ and the CVS decomposition is performed with a threshold $\epsilon_{T}$ based on the instantaneous enstrophy $Z(t)$ using (2.3). We then determine the index set of the filtered wavelet basis, corresponding to the wavelet coefficients that are larger than this threshold. In order to account for the translation of coherent vortices and the creation of finer scales that may not be captured by the filtered wavelet basis, this index set is expanded by adding neighbour wavelets in space and scale, comprising the socalled security zone (figure 16). It includes one neighbour in each space direction and the wavelets at the next smaller scale having the same position. This security zone width is sufficient because the CFL criterion ensures that information does not travel faster than $\Delta x / \Delta t$ ( $\Delta x$ being the smallest space step and $\Delta t$ the time step). Also, the addition of the wavelets of the next octave prevents aliasing errors since the Navier-Stokes equations have a quadratic nonlinearity. The adaptive wavelet basis, which corresponds to the filtered wavelet basis supplemented with the security zone, fully retains the nonlinear dynamics of the flow and tracks its evolution in space and scale. The security zone concept has been introduced by Liandrat \& Tchamitchian (1990) for computing solutions to the one-dimensional Burgers equation and has been used by Fröhlich \& Schneider (1999) to compute solutions to the two-dimensional Navier-Stokes equations on an adaptive wavelet basis.

Finally, the solution is advanced in time and only the modes belonging to the adaptive wavelet basis are retained. The coherent vorticity is reconstructed by an inverse wavelet transform and the corresponding coherent velocity is computed using Biot-Savart's relation (2.5). Discarding the incoherent enstrophy at each time step is equivalent to turbulent dissipation because the incoherent flow is structureless and decorrelated, corresponding to a kind of thermal background noise. 


\subsection{Dynamical comparison between DNS and CVS}

Two simulations, one DNS and one CVS, have been performed at a resolution of $N=128^{3}$. The mean streamwise velocity profile employed as an initial condition is $U(y)=\Delta U \tanh \left(2 y / \delta_{m}^{0}\right)$, where $\delta_{m}^{0}$ has been chosen such that four KelvinHelmholtz vortices develop when the profile is perturbed. To trigger this instability we superimpose Gaussian noise in the vortical region. At later times, three-dimensional instabilities appear and streamwise ribs are formed.

The beginning of the simulation corresponds to the initiation of a transition towards turbulence. Up to $t=7 \delta_{m}^{0} / \Delta U$, the two-dimensional Kelvin-Helmholtz instability develops in the streamwise direction, creating four two-dimensional rolls. During this first phase, there is no vortex stretching and therefore no production of enstrophy, which is actually slowly dissipated (cf. figure $20 b$ ). Then three-dimensional instabilities begin to deform the rolls in the spanwise direction. As these instabilities are in phase opposition for each pair of rolls, they are brought together and a first pairing occurs at time $t=9 \delta_{m}^{0} / \Delta U$ (figure 17). After this point, vortex stretching begins and gives rise to the formation of vortex ribs in between the rolls, resulting in the production of enstrophy as observed in (figure 20b). At $t=18 \delta_{m}^{0} / \Delta U$, a second pairing occurs, more and more entangled vortices are generated around the two main rolls, and the mixing layer becomes turbulent. As a result, hairpin vortices are formed and stretched by velocity gradients, and the production of enstrophy becomes more pronounced (figure 18). At $t=25 \delta_{m}^{0} / \Delta U$, the enstrophy production saturates as observed in (figure 18) and the vorticity field consists of a complex mixture of vortex tubes (figure 19).

Before performing quantitative analyses of the differences between DNS and CVS, we study the vorticity fields in physical space. For this we plot the iso-surfaces of the vorticity modulus in figures 17,18 , and 19 at $t=9 \delta_{m}^{0} / \Delta U, t=18 \delta_{m}^{0} / \Delta U$, and $t=$ $25 \delta_{m}^{0} / \Delta U$, respectively. Until the second pairing occurs at $t=18 \delta_{m}^{0} / \Delta U$, no differences are observed between the DNS and the CVS (figure 18). The flow is not yet fully turbulent, thus the retained wavelet modes describe the flow completely. After the transition to turbulence, the situation changes and the CVS-filtered computation exhibits the structure and topology of the vortex tubes more clearly than the DNS (figure 19) because the random fluctuations of the incoherent vorticity have been filtered out. Nevertheless, all organized vortex tubes are still present, whatever their scale and intensity.

\subsection{Statistical comparison between DNS and CVS}

This correspondence between CVS and DNS flows is quantified by studying the time evolution of integral quantities, like energy and enstrophy. Figure 20 shows that, before the flow is fully turbulent, both energy and enstrophy are the same for CVS and DNS. After the flow has become fully turbulent (after about $t=18 \delta_{m}^{0} / \Delta U$ ), the nonlinear flow dynamics begins to produce incoherent enstrophy. The CVS filtering removes this incoherent enstrophy and this explains why the CVS flow contains less enstrophy than the DNS flow. Contrary to standard approaches in turbulence modelling, with CVS there is no need to adjust a turbulent viscosity parameter because the threshold is automatically set by the instantaneous enstrophy, as explained in $\S 2$.

On the other hand, the time evolution of large-scale statistics such as turbulent kinetic energy $E$ is almost the same for CVS and DNS; up to the final time the discrepancy remains less than $0.4 \%$ of $E$. This is a result of the noise-like nature of the incoherent vorticity, which contributes almost nothing to the total energy because it is cancelled out in the Biot-Savart kernel (equation (2.5)). In contrast, the coherent 

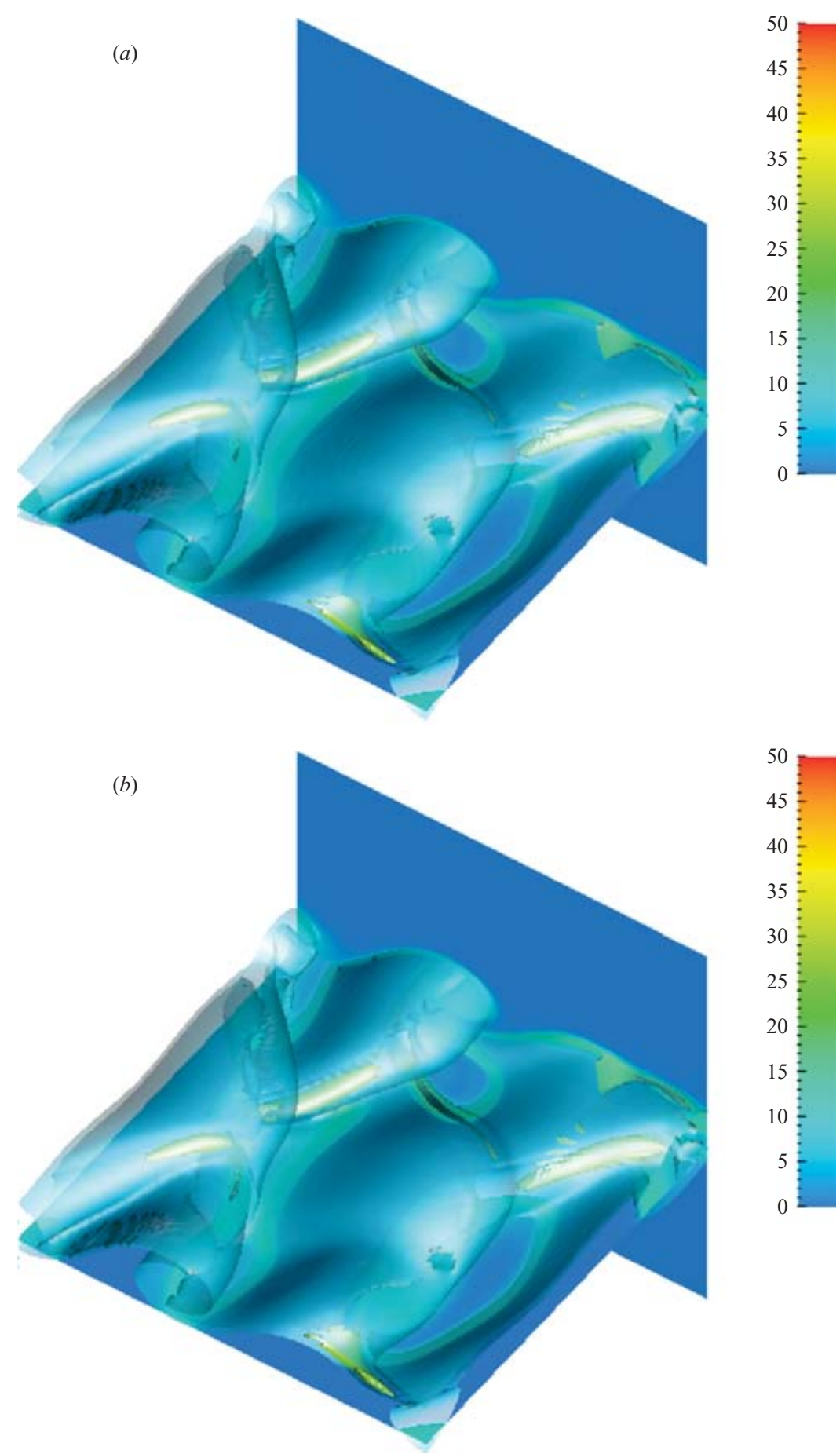

FIGURE 17. Comparison between DNS $(a)$ and CVS $(b)$. Iso-surfaces of the vorticity modulus coloured by the spanwise vorticity at $t=9 \delta_{m}^{0} / \Delta U$. 


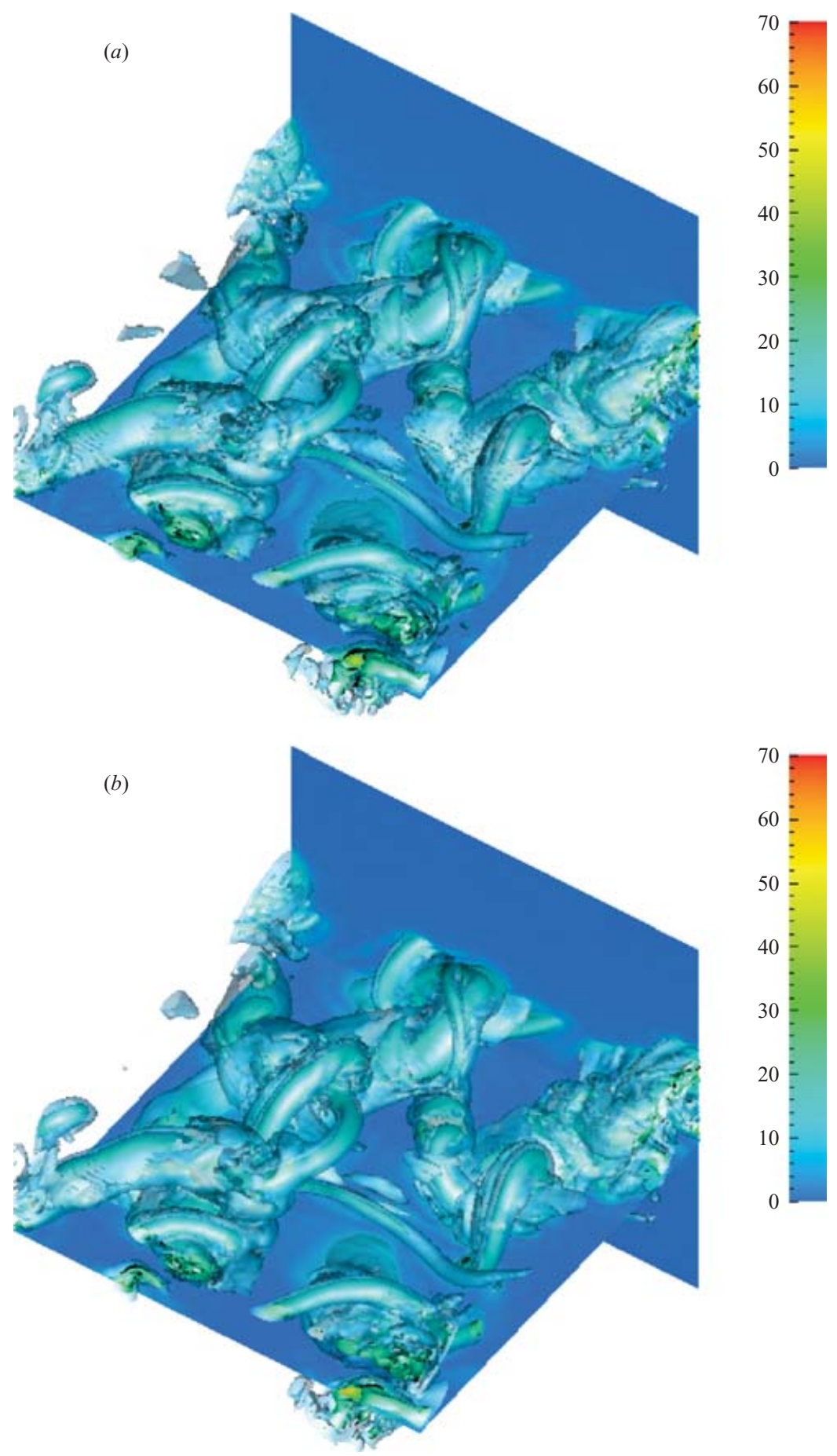

FIGURE 18. Comparison between DNS $(a)$ and CVS $(b)$. Iso-surfaces of the vorticity modulus coloured by the spanwise vorticity at $t=18 \delta_{m}^{0} / \Delta U$. 

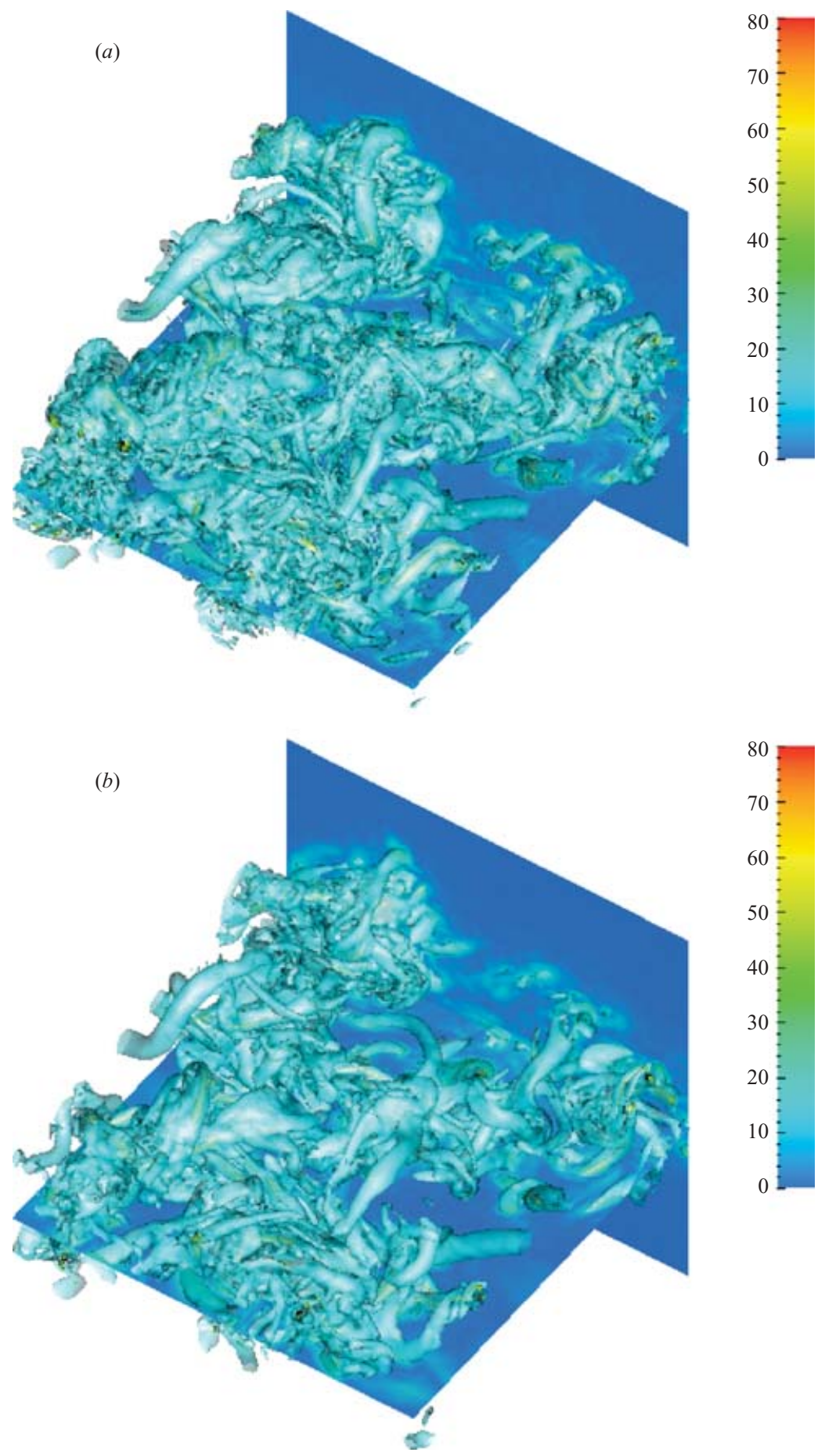

FIGURE 19. Comparison between DNS $(a)$ and CVS $(b)$. Iso-surfaces of the vorticity modulus coloured by the spanwise vorticity at $t=25 \delta_{m}^{0} / \Delta U$. 

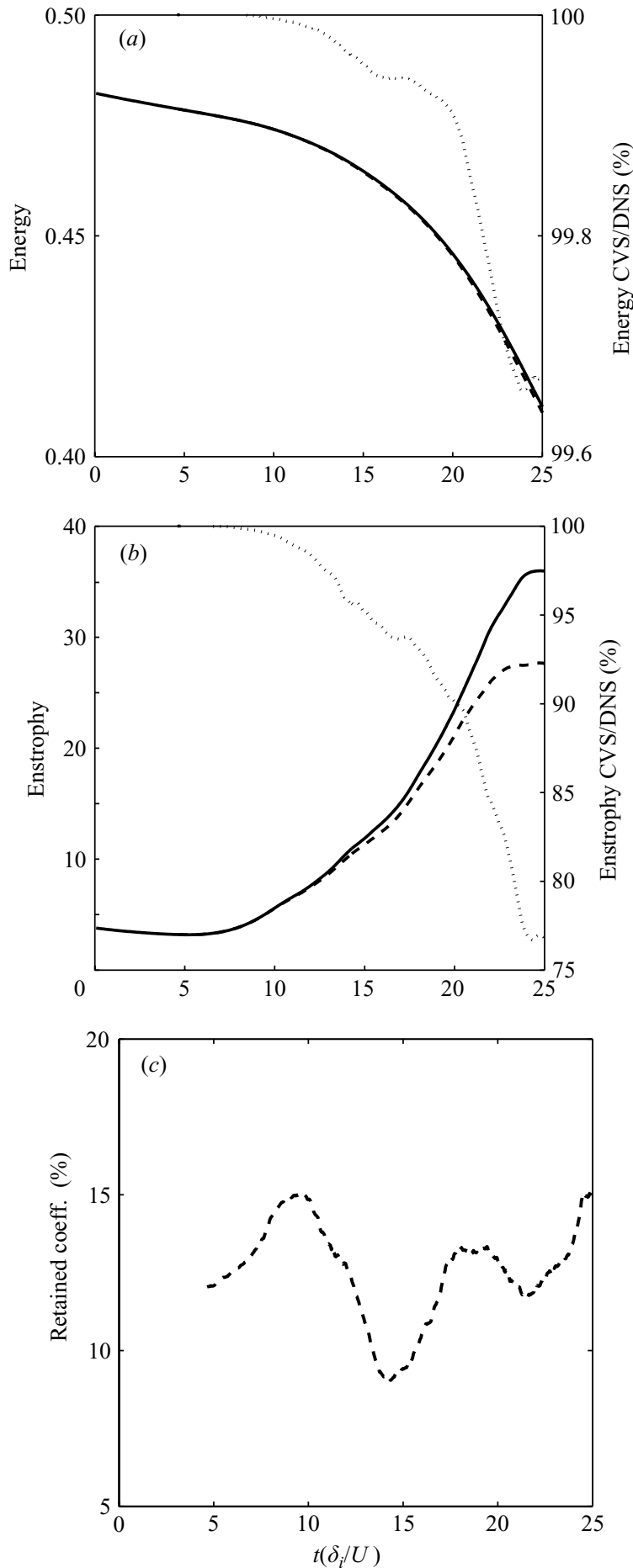

Figure 20. (a) Time evolution of energy. (b) Time evolution of enstrophy. We compare DNS (solid line) and CVS (dashed line), and also plot the ratio CVS/DNS (dotted line). (c) Time evolution of the percentage of retained wavelet coefficients (including the security zone) used by CVS. Note that the first two peaks correspond to the times when vortex pairing is occurring. 

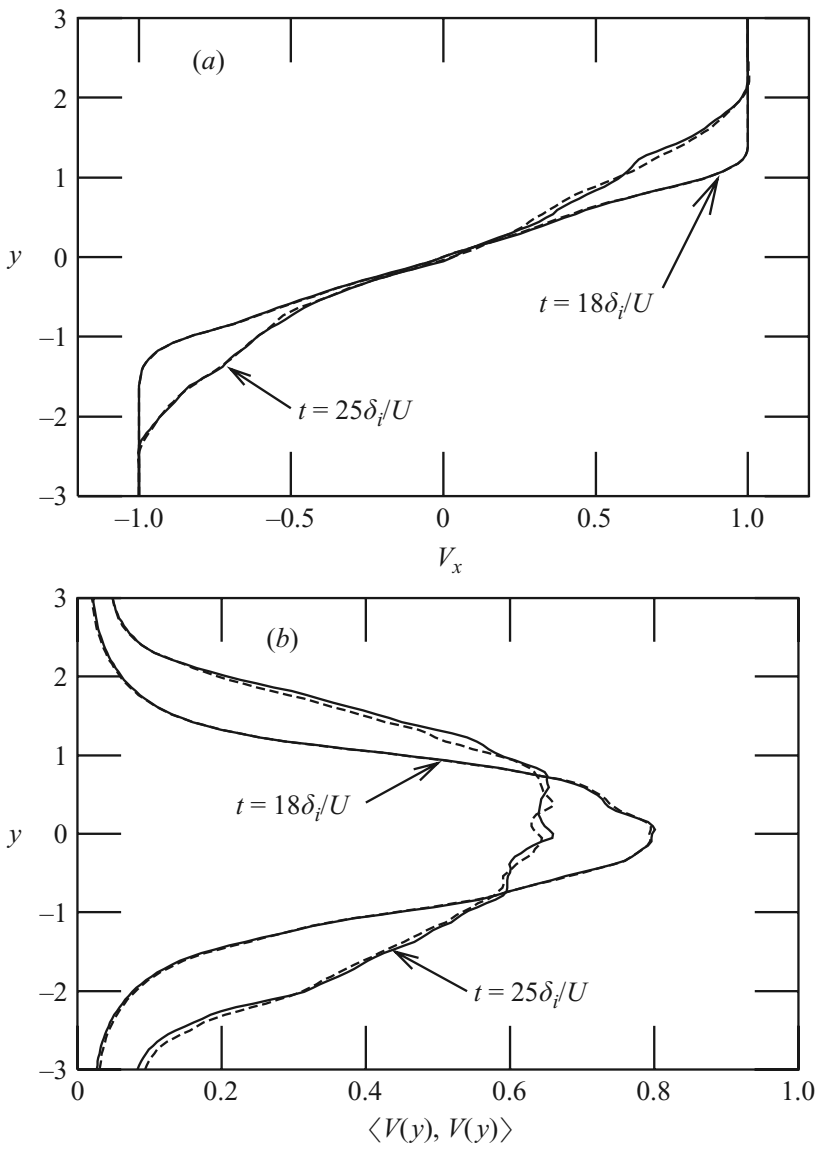

FIGURE 21. Comparison between CVS (dashed line) and DNS (solid line). Cross-stream profile of mean $(a)$ and variance $(b)$ of the streamwise velocity at time $t=18 \delta_{m}^{0} / \Delta U$ and $t=25 \delta_{m}^{0} / \Delta U$.

vortex tubes are localized and induce a coherent velocity that accounts for virtually all of the energy.

In figure $20(c)$ we present the number of wavelet modes retained during CVS, which varies between $8 \%$ and $15 \%$ of $N$ corresponding to a factor of about 2 in each Cartesian direction. About $4 \%$ of $N$ are in the filtered wavelet basis and the rest correspond to the security zone, which has been added to track the nonlinear dynamics in space and scale. This figure shows that the number of active wavelet coefficients evolves in time. In particular, when pairings occur the number of wavelets increases, as reflected in the first two peaks in figure $20(c)$.

In figure 21 we analyse the cross-stream profile of the mean and variance of the streamwise velocity at times $t=18 \delta_{m}^{0} / \Delta U$ and $t=25 \delta_{m}^{0} / \Delta U$. The agreement between CVS and DNS at both times is excellent. Similar agreement is observed for the crossstream profiles of vorticity fluctuation variances at time $t=18 \delta_{m}^{0} / \Delta U$ (figure 22), but not at the later time $t=25 \delta_{m}^{0} / \Delta U$, when the flow has become fully turbulent. At this time, the CVS method is filtering out part of the vorticity fluctuations and therefore the profile obtained with CVS is slightly narrower than that obtained with DNS. In figure 23 we present one-dimensional streamwise energy spectra for each of the three velocity components. During the simulation, energy is transferred from large 


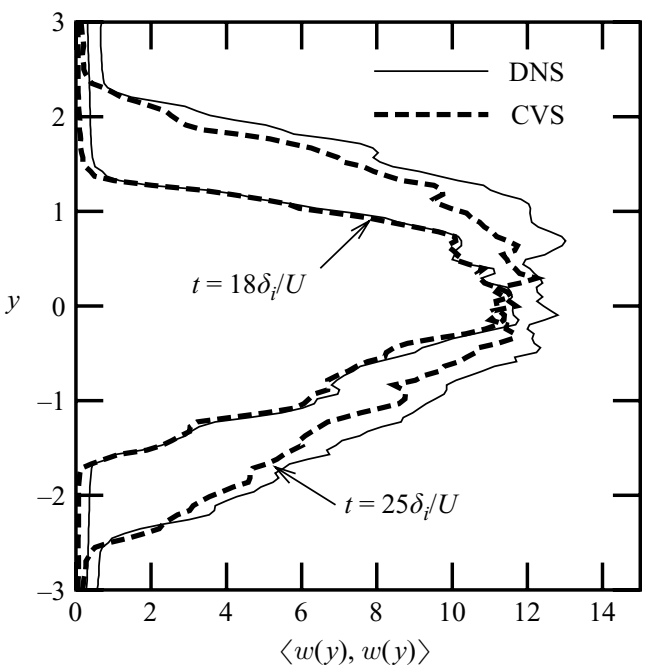

Figure 22. Comparison between CVS (dashed line) and DNS (solid line). Cross-stream profile of the vorticity variance averaged in the horizontal plane at time $t=18 \delta_{m}^{0} / \Delta U$ and $t=25 \delta_{m}^{0} / \Delta U$.

scales to small scales and a self-similar $k^{-5 / 3}$ inertial subrange begins to develop. The comparison between CVS and DNS confirms the result of the CVS filtering found in $\S 3$, namely, the energy spectra for CVS are the same as for DNS at large scales, whereas at small scales the filtering discards the incoherent enstrophy which is equivalent to turbulent dissipation.

We now consider the PDF of the vorticity. At $t=18 \delta_{m}^{0} / \Delta U$, the PDFs of the spanwise and streamwise vorticity are highly non-Gaussian and slightly skewed due to the presence of ribs and rolls. The turbulence intensity increases and by $t=25 \delta_{m}^{0} / \Delta U$ the PDFs have become more symmetric, although their flatness remains high. We also observe that the maximum values of vorticity have increased due to the production of enstrophy by vortex stretching. The PDFs of both CVS and DNS remain very similar up to the end of the simulations. This, together with the flow visualizations (figures 17, 18, and 19), demonstrates that CVS preserves the structures present in the flow and their nonlinear dynamics. The only difference observed is that the vorticity field computed by CVS is cleaner (figure 19b) than the one computed by DNS (figure 19a) because the fuzziness in the latter has been removed by filtering the incoherent vorticity.

\section{Conclusions and perspectives}

In this paper we have assessed the potential of the CVS method as a simulation tool for three-dimensional turbulent mixing layers. The CVS filtering extracts the coherent structures from turbulent flows using an orthogonal wavelet basis. The coherent vorticity is reconstructed from the few wavelet coefficients whose modulus is larger than a threshold, which depends only on the resolution and on the total enstrophy, while the remaining wavelet coefficients are discarded to model turbulent dissipation. Then the induced coherent velocity is computed using Biot-Savart's relation.

We have first applied this method to two three-dimensional time-developing mixing layers, forced and unforced, at a given time instant. The coherent flow is represented 

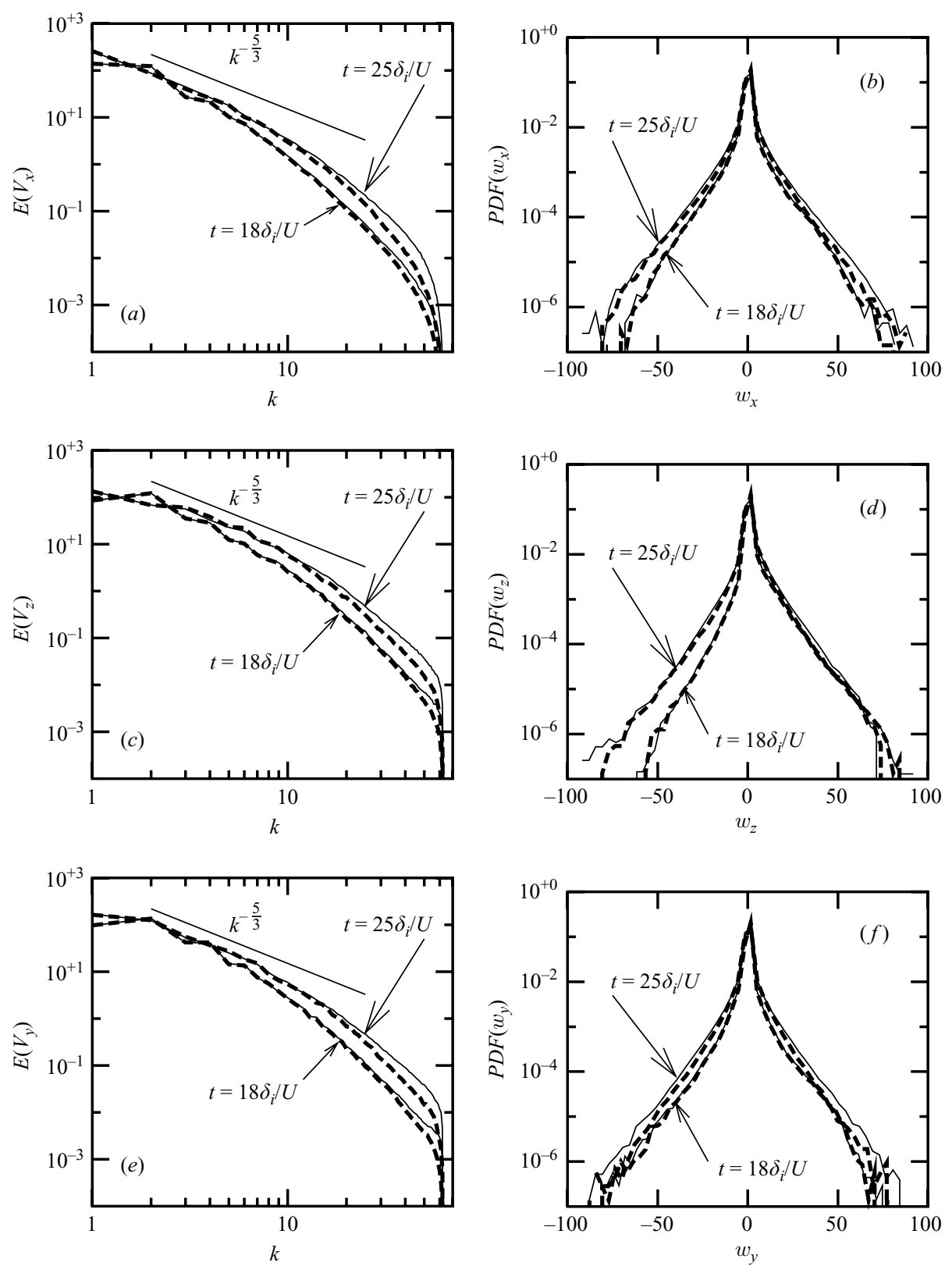

FIGURE 23. Comparison between CVS (dashed line) and DNS (solid line) at time $t=18 \delta_{m}^{0} / \Delta U$ and $t=25 \delta_{m}^{0} / \Delta U .(a, c, e)$ : One-dimensional energy spectra in the streamwise direction for the streamwise $(a)$, spanwise $(c)$ and vertical $(e)$ components of velocity. $(b, d, f)$ : PDFs of the streamwise $(b)$, spanwise $(d)$, and vertical $(f)$ components of vorticity.

by only about $4 \%$ of the wavelet coefficients, and yet retains all the essential characteristics of the total flow. It contains most of the energy and enstrophy, preserves the non-Gaussianity of the vorticity PDF, and has the same spectral distribution of energy. We have also checked that the associated incoherent flow is structureless, decorrelated, and exhibits a much narrower vorticity PDF. 
The advantage of CVS filtering in comparison to LES filtering has also been demonstrated for turbulent mixing layers. The incoherent vorticity discarded by CVS is structureless, while the subgrid-scale vorticity associated with LES is not, since it contains the small scales of the coherent structures. The vorticity PDF is much narrower for the CVS-filtered than for the LES-filtered flow. Moreover, the incoherent flow exhibits a flat energy spectrum and this decorrelation suggests that it has reached a kind of equilibrium.

To check the influence of the CVS filtering on the flow dynamics we performed several time integrations using the filtered fields as initial conditions. The results confirmed that the statistics of the CVS-filtered flow remain close to those of the total flow.

We then performed other simulations in which, at each time step, we applied CVS filtering and added a security zone to provide room for the coherent structures to move and distort. For this, we coupled the CVS filtering with a DNS code and performed a CVS, however saving neither memory nor CPU time, as would be the case if one used an adaptive discretization. The results demonstrate that CVS preserves the nonlinear dynamics of the flow, in particular the formation of primary two-dimensional vortices and secondary three-dimensional ribs, although it eliminates the incoherent enstrophy which corresponds to turbulent dissipation. We observe that CVS results in similar statistics, such as mean velocity and velocity variance, energy spectra, and vorticity PDF, as DNS.

Work in progress deals with the development of a CVS code to solve the threedimensional Navier-Stokes equations in an adaptive wavelet basis. This combines an Eulerian representation of the flow and a Lagrangian adaptation strategy for the computed modes, which are remapped at each time step using CVS filtering. The dynamical adaptation of the grid in physical space, which is inherent in CVS, allows the nonlinearly active coherent flow to be evolved with a reduced number of computed modes, while the passive incoherent flow is eliminated to model turbulent dissipation. We have already shown that CVS yields results similar to those obtained with DNS for different kinds of two-dimensional flows: homogeneous isotropic turbulent flows (Fröhlich \& Schneider 1999), temporally developing mixing layers (Schneider \& Farge 2000; Farge \& Schneider 2001), and flows past a cylinder (Schneider \& Farge 2002). For three-dimensional reaction-diffusion equations we have shown (Roussel et al. 2003) that adaptive wavelet computation results in significant reduction in memory requirements and CPU time. The first results for three-dimensional compressible flows using this adaptive wavelet solver are promising and will be reported elsewhere.

Part of this work was performed during the 2000 Center for Turbulence Research Summer Program. M. F. and K. S. gratefully acknowledge financial support from the Center for Turbulence Research, Stanford University, and the NSF-CNRS-DAAD joint research program (contract 9201). G. P. was supported by the Commission of the European Communities Contract FMRX-CT98-0184 TMR Project "Wavelets in Numerical Simulation". We also thank Pierre Comte for providing his spectral code and for fruitful discussions.

\section{REFERENCES}

Brown, G. L. \& Roshko, A. 1974 On density effects and large structures in turbulent mixing layers. J. Fluid Mech. 64, 775-816. 
Comte, P., Lesieur, M. \& Lamballais, E. 1992 Large- and small-scale stirring of vorticity and passive scalar in a 3-d temporal mixing layer. Phys. Fluids A 4, 2761-2778.

Daubechies, I. 1992 Ten Lectures on Wavelets. SIAM, CBMS-NSF Conference Series in Applied Mathematics, vol. 61. Philadelphia.

DonoHO, D. 1993 Unconditional bases are optimal bases for data compression and statistical estimation. Appl. Comput. Harmon. Anal. 1, 100-115.

Donoho, D. \& Johnstone, I. 1994 Ideal spatial adaption via wavelet shrinkage. Biometrica 81, 425-455.

FARGE, M. 1992 Wavelet transforms and their applications to turbulence. Annu. Rev. of Fluid Mech. 24, 395-457.

Farge, M., Pellegrino, G. \& Schneider, K. 2001 Coherent vortex extraction in 3D turbulent flows using orthogonal wavelets. Phys. Rev. Lett. 87(5), 45011-45014.

Farge, M. \& Schneider, K. 2001 Coherent Vortex Simulation (CVS), a semi-deterministic turbulence model using wavelets. Flow, Turbulence Combust. 66, 393-426.

Farge, M., Schneider, K. \& Kevlahan, N. 1999 Non-Gaussianity and Coherent Vortex Simulation for two-dimensional turbulence using an adaptive orthonormal wavelet basis. Phys. Fluids 11, 2187-2201.

Farge, M., Schneider, K., Pellegrino, P., Wray, A. \& Rogallo, B. 2003 Coherent vortex extraction in three-dimensional homogeneous turbulence: Comparison between CVS-wavelet and POD-Fourier decompositions. Phys. Fluids 15, 2886-2896.

Fröhlich, J. \& SCHNEIDER, K. 1999 Computation of decaying turbulence in an adaptive wavelet basis. Physica D 134, 337-361.

Ho, C.-M. \& Huerre, P. 1984 Perturbed free shear layers. Annu. Rev. Fluid. Mech. 16, 365-424.

LEMARIÉ, P. G. 1992 Analyses multirésolutions non orthogonales, commutation entre projecteurs de dérivation et ondelettes à divergence nulle. Revista Mat. Iberoamericana 8, 221-236.

Liandrat, J. \& TCHAmitchian, P. 1990 Resolution of the 1D regularized Burgers equation using a spatial wavelet approximation: algorithm and numerical results. ICASE Rep. 90-83.

Rogers, M. \& Moser, R. 1994 Direct simulation of a self-similar turbulent mixing layer. Phys. Fluids 6, 903-923.

Roussel, O., Schneider, K., Tsigulin, A. \& Bockhorn, H. 2003 A conservative fully adaptive multiresolution algorithm for parabolic PDEs. J. Comput. Phys. 188, 493-523.

SCHNEIDER, K. \& FARGE, M. 2000 Numerical simulation of temporally growing mixing layer in an adaptive wavelet basis. C. R. Acad. Sci. Paris II b, 328, 263-269.

SchneIder, K. \& FARge, M. 2002 Adaptive wavelet simulation of a flow around an impulsively started cylinder using penalisation. Appl. Comput. Harm. Anal. 12, 374-380.

Winckelmans, G. S. 1995 Some progress in large-eddy simulation using the 3D vortex particle method. CTR Annual Research Briefs, pp. 391-415. 